ARTICLE

\title{
Fonio millet genome unlocks African orphan crop diversity for agriculture in a changing climate
}

Michael Abrouk (10) 1,14, Hanin Ibrahim Ahmed ${ }^{1,14}$, Philippe Cubry (1D 2,14, Denisa Šimoníková3 ${ }^{2}$ Stéphane Cauet (D) 4 , Yveline Pailles', Jan Bettgenhaeuser (10 1, Liubov Gapa (1) ${ }^{1}$, Nora Scarcelli², Marie Couderc², Leila Zekraoui², Nagarajan Kathiresan ${ }^{5}$, Jana Čížková3 ${ }^{3}$ Eva Hřibová ${ }^{3}$, Jaroslav Doležel (D) ${ }^{3}$, Sandrine Arribat ${ }^{4}$, Hélène Bergès ${ }^{4,6}$, Jan J. Wieringa (1) ${ }^{7}$, Mathieu Gueye ${ }^{8}$, Ndjido A. Kane (1) 9,10, Christian Leclerc ${ }^{11,12}$, Sandrine Causse ${ }^{11,12}$, Sylvie Vancoppenolle ${ }^{11,12}$, Claire Billot ${ }^{11,12}$, Thomas Wicker ${ }^{13}$, Yves Vigouroux (D) ${ }^{2}$, Adeline Barnaud (D) ${ }^{2,10 凶} \&$ Simon G. Krattinger (1D ${ }^{1 凶}$

Sustainable food production in the context of climate change necessitates diversification of agriculture and a more efficient utilization of plant genetic resources. Fonio millet (Digitaria exilis) is an orphan African cereal crop with a great potential for dryland agriculture. Here, we establish high-quality genomic resources to facilitate fonio improvement through molecular breeding. These include a chromosome-scale reference assembly and deep re-sequencing of 183 cultivated and wild Digitaria accessions, enabling insights into genetic diversity, population structure, and domestication. Fonio diversity is shaped by climatic, geographic, and ethnolinguistic factors. Two genes associated with seed size and shattering showed signatures of selection. Most known domestication genes from other cereal models however have not experienced strong selection in fonio, providing direct targets to rapidly improve this crop for agriculture in hot and dry environments.

\footnotetext{
${ }^{1}$ Center for Desert Agriculture, Biological and Environmental Science \& Engineering Division (BESE), King Abdullah University of Science and Technology (KAUST), Thuwal, Saudi Arabia. ${ }^{2}$ DIADE, Univ Montpellier, IRD, Montpellier, France. ${ }^{3}$ Institute of Experimental Botany of the Czech Academy of Sciences, Centre of the Region Hana for Biotechnological and Agricultural Research, Olomouc, Czech Republic. ${ }^{4}$ CNRGV Plant Genomics Center, INRAE,

Toulouse, France. ${ }^{5}$ Supercomputing Core Lab, King Abdullah University of Science and Technology (KAUST), Thuwal, Saudi Arabia. ${ }^{6}$ Inari Agriculture, One Kendall Square Building 600/700, Cambridge, MA 02139, USA. ${ }^{7}$ Naturalis Biodiversity Center, Leiden, the Netherlands. ${ }^{8}$ Laboratoire de Botanique, Département de Botanique et Géologie, IFAN Ch. A. Diop/UCAD, Dakar, Senegal. ${ }^{9}$ Senegalese Agricultural Research Institute, Dakar, Senegal. ${ }^{10}$ Laboratoire Mixte International LAPSE, Dakar, Senegal. ${ }^{11}$ CIRAD, UMR AGAP, Montpellier, France. ${ }^{12}$ AGAP, Université de Montpellier, Cirad, INRAE, Institut Agro, Montpellier, France. ${ }^{13}$ Department of Plant and Microbial Biology, University of Zurich, Zürich, Switzerland. ${ }^{14}$ These authors contributed equally: Michael Abrouk, Hanin Ibrahim Ahmed, Philippe Cubry. ${ }^{凶}$ email: adeline.barnaud@ird.fr; simon.krattinger@kaust.edu.sa
} 
H umanity faces the unprecedented challenge of having to sustainably produce healthy food for 9-10 billion people by 2050 in a context of climate change. A more efficient use of plant diversity and genetic resources in breeding has been recognized as a key priority to diversify and transform agriculture $^{1-3}$. The Food and Agriculture Organization of the United Nations (FAO) stated that arid and semi-arid regions are the most vulnerable environments to increasing uncertainties in regional and global food production ${ }^{4}$. In most countries of Africa and the Middle East, agricultural productivity will decline in the near future ${ }^{4}$, because of climate change, land degradation, and groundwater depletion ${ }^{5}$. Agricultural selection, from the early steps of domestication to modern-day crop breeding, has resulted in a marked decrease in agrobiodiversity ${ }^{6,7}$. Today, three cereal crops alone, bread wheat (Triticum aestivum), maize (Zea mays), and rice (Oryza sativa) account for more than half of the globally consumed calories ${ }^{8}$.

Many of today's major cereal crops, including rice and maize, originated in relatively humid tropical and sub-tropical regions $s^{9,10}$. Although plant breeding has adapted the major cereal crops to a wide range of climates and cultivation practices, there is limited genetic diversity within these few plant species for cultivation in the most extreme environments. On the other hand, crop wild relatives and orphan crops are often adapted to extreme environments and their utility to unlock marginal lands for agriculture has recently regained interest $2,6,11-14$. Current technological advances in genomics and genome editing provide an opportunity to rapidly domesticate wild relatives and to improve orphan crops ${ }^{15,16}$. De novo domestication of wild species or rapid improvement of semi-domesticated crops can be achieved in less than a decade by targeting a few key genes ${ }^{6}$.

White fonio (Digitaria exilis (Kippist) Stapf) (Fig. 1) is an indigenous West African millet species with a great potential for agriculture in marginal environments ${ }^{17,18}$. Fonio is a small annual herbaceous $\mathrm{C} 4$ plant, which produces very small $(\sim 1 \mathrm{~mm})$ grains that are tightly surrounded by a husk ${ }^{19}$ (Fig. 1). Fonio is cultivated under a large range of environmental conditions, from a tropical monsoon climate in western Guinea to a hot, arid desert climate in the Sahel zone. Some extra-early

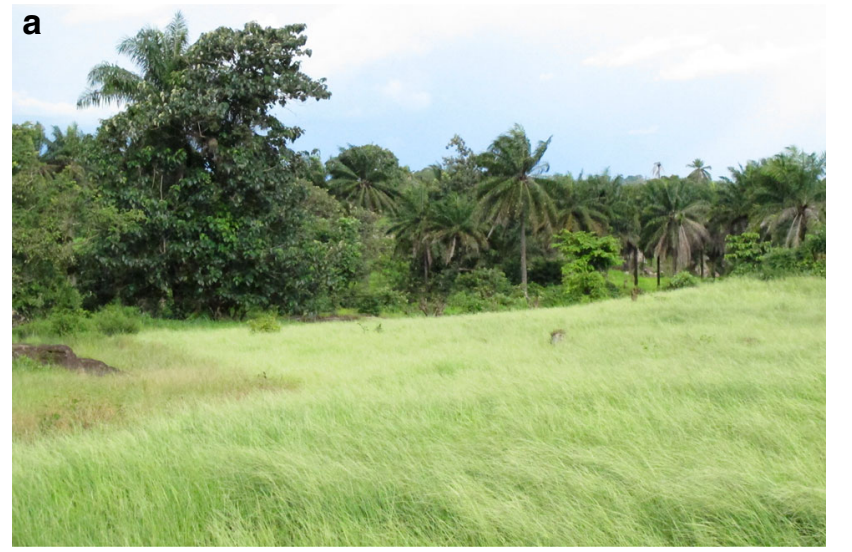

b

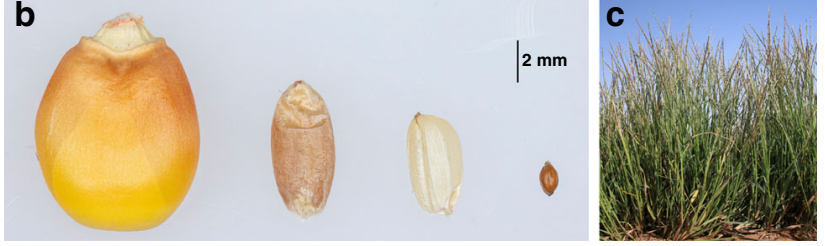

Fig. 1 Phenotype of fonio (Digitaria exilis). a Field of cultivated fonio in Guinea. b Grains of maize, wheat, rice, and fonio (from left to right). c Plants of the fonio accession CM05836. maturing fonio varieties produce mature grains in only 70-90 days ${ }^{19}$, which makes fonio one of the fastest maturing cereals. Because of its quick maturation, fonio is often grown to avoid food shortage during the lean season (period before main harvest), which is why fonio is also referred to as 'hungry rice'. In addition, fonio is drought tolerant and adapted to nutrient-poor, sandy soils ${ }^{20}$. Despite its local importance for agriculture in West Africa, fonio shows many unfavorable characteristics that resemble undomesticated plants: residual seed shattering, lodging, and lower yields than other cereals ${ }^{18}$ (Supplementary Fig. 1). The most likely wild progenitor of cultivated fonio is the tetraploid annual weed $D$. longiflora that is widely distributed in tropical Africa. It has been suggested that fonio was domesticated more than 5000 years ago in the Inner Niger Delta of central Mali. Compared to $D$. exilis, the wild D. longiflora shows even heavier seed shattering and hairy spikelets, which suggests that some selection for reduced seed shattering and spikelet hairiness occurred in fonio ${ }^{21}$. In the past few years, fonio has gained in popularity inside and outside of West Africa because of its nutritional qualities.

Here, we present the establishment of a comprehensive set of genomic resources for fonio, which constitutes the first step towards harnessing the potential of this cereal crop for agriculture in harsh environments. These resources include the generation of a high-quality, chromosome-scale reference assembly and the deep re-sequencing of a diversity panel that includes wild and cultivated accessions covering a wide geographic range.

\section{Results}

Chromosome-scale fonio reference genome assembly. Fonio is a tetraploid species $(2 n=4 \times=36)^{22}$ with a highly inbreeding reproductive system ${ }^{17}$. To build a $D$. exilis reference assembly, we chose an accession from one of the driest regions of fonio cultivation, CM05836 from the Mopti region in Mali. The size of the CM05836 genome was estimated to be $893 \mathrm{Mb} / 1 \mathrm{C}$ by flow cytometry (Supplementary Figs. 2 and 3), which is in line with previous reports ${ }^{22}$. The CM05836 genome was sequenced and assembled using deep sequencing of multiple short-read libraries (Supplementary Table 1), including Illumina paired-end (321fold coverage), mate-pair (241-fold coverage) and linked-read (10× Genomics, 84-fold coverage) sequencing. The raw reads were assembled and scaffolded with the software package DeNovoMAGIC3 (NRGene), which has recently been used to assemble various high-quality plant genomes ${ }^{23-25}$. Integration of $\mathrm{Hi}-\mathrm{C}$ reads (122-fold coverage, Supplementary Table 2) and a Bionano optical map (Supplementary Table 3) resulted in a chromosome-scale assembly with a total length of 716,471,022 bp, of which $\sim 91.5 \%(655,723,161 \mathrm{bp})$ were assembled in 18 pseudomolecules. A total of $60.75 \mathrm{Mb}$ were unanchored (Table 1). Of 1440 Embryophyta single copy core genes (BUSCO v3.0.2), 96.1\% were recovered in the CM05836 assembly, $2.9 \%$ were missing, and $1 \%$ was fragmented. As no genetic $D$. exilis map is available, we used chromosome painting to further assess the quality of the CM05836 assembly. Pools of short oligonucleotides covering each one of the 18 pseudomolecules were designed based on the CM05836 assembly, fluorescently labeled, and hybridized to mitotic metaphase chromosome spreads of CM05836 ${ }^{26}$. Each of the 18 libraries specifically hybridized to only one chromosome pair, confirming that our assembly unambiguously distinguished homoeologous chromosomes (Fig. 2a, Supplementary Fig. 4). Centromeric regions contained a tandem repeat with a $314 \mathrm{bp}$ long unit, which was found in all fonio chromosomes (Supplementary Fig. 5). We also re-assembled all the data with the opensource TRITEX pipeline ${ }^{27}$ and the two assemblies showed a high degree of collinearity (Supplementary Fig. 6). 


\begin{tabular}{|ll|}
\hline $\begin{array}{l}\text { Table } 1 \text { Statistics of the fonio genome assembly and } \\
\text { annotation. }\end{array}$ \\
& CM05836 \\
\hline & 701.662 \\
Length of DeNovoMAGIC3 assembly (Mb) & 8457 \\
Number of scaffolds & 10.741 \\
N50 (Mb) & 1.009 \\
N90 (Mb) & 716.471 a \\
Length of genome assembly (Mb) & 655.723 \\
Total length of pseudomolecules (Mb) & 18,026 \\
Number of anchored contigs & 83.702 \\
N50 of anchored contigs (kb) & $17.001(2.6 \%)$ \\
Gap size (Mb) & 57,021 \\
Number of genes & 60.748 \\
Total length of unanchored chromosomes (Mp) & 11,129 \\
Number of unanchored contigs & 9.191 \\
N50 of unanchored contigs (kb) & $2.962(4.9 \%)$ \\
Gap size (Mb) & 2821 \\
Number of genes & \\
BUSCO & 96.1 \\
Complete (\%) & 84.3 \\
Duplicated (\%) & 1 \\
Fragmented (\%) & 2.9 \\
Missing (\%) & \\
\hline
\end{tabular}

aDeNovoMAGIC3 + Hi-C + optical map

We compared the fonio pseudomolecule structure to foxtail millet (Setaria italica; $2 n=2 \times=18$ ), a diploid relative with a fully sequenced genome ${ }^{28}$. The fonio genome shows a syntenic relationship with the genome of foxtail millet, with two homoeologous sets of nine fonio chromosomes (Supplementary Fig. 7). Without a clear diploid ancestor, we could not directly disentangle the two sub-genomes based on genomic information from diploid ancestors ${ }^{29}$. We thus used a genetic structure approach based on full-length long terminal repeat retrotransposons (fl-LTR-RT) as an alternative strategy. A total of $11 \mathrm{fl-LTR-}$ RT families with more than 30 elements were identified and defined as a 'populations', allowing us to apply genetic structure analyses that are often used in population genomics ${ }^{30}$. We searched for fl-LTR-RT clusters that only appeared in one of the two homoeologous sub-genomes. Out of the 11 fl-LTR-RT populations analyzed, two allowed us to discriminate the subgenomes (Fig. 2b, Supplementary Fig. 8). The two families belong to the Gypsy superfamily and dating of insertion time was estimated to be $\sim 1.56$ million years ago (MYA) (58 elements, 0.06-4.67 MYA) and 1.14 MYA (36 elements, 0.39-1.96 MYA), respectively. The two putative sub-genomes were designated A and $\mathrm{B}$ and chromosome numbers were assigned based on the synteny with foxtail millet (Supplementary Fig. 7).

Gene annotation was performed using the MAKER pipeline (v3.01.02) with $34.1 \%$ of the fonio genome masked as repetitive. Transcript sequences of CM05836 from flag leaves, grains, panicles, and whole above-ground seedlings (Supplementary Table 4) in combination with protein sequences of publicly available plant genomes were used to annotate the CM05836 assembly. This resulted in the annotation of 59,844 protein-coding genes (57,023 on 18 pseudomolecules and 2821 on unanchored chromosome) with an average length of $2.5 \mathrm{~kb}$ and an average exon number of 4.6. The analysis of the four CM05836 RNA-seq samples showed that 44,542 protein coding genes $(74.3 \%)$ were expressed $(>0.5$ transcripts per million), which is comparable to the annotation of the bread wheat genome (Supplementary Table 5) ${ }^{31,32}$.

Synteny with other cereals. Whole genome comparative analyses of the CM05836 genome with other grass species were consistent with the previously established phylogenetic relationships of fonio $^{20}$. A comparison of the CM05836 A and B sub-genomes identified a set of 16,514 homoeologous gene pairs that fulfilled the criteria for evolutionary analyses (Fig. 2c and Supplementary Data 1). The estimation of synonymous substitution rates (Ks) among homoeologous gene pairs revealed a divergence time of the two sub-genomes of roughly 3 MYA (Supplementary Fig. 9 and Supplementary Data 1). These results indicate that D. exilis is a recent allotetraploid species. A Ks distribution using orthologous genes revealed that fonio diverged from the other members of the Paniceae tribe (broomcorn millet (Panicum miliaceum), Hall's panicgrass ( $P$. hallii), and foxtail millet (S. italica)) between 14.6 and 16.9 MYA, and from the Andropogoneae tribe (sorghum (Sorghum bicolor) and maize (Z. mays)) between 21.5 and 26.9 MYA. Bread wheat (T. aestivum), goatgrass (Aegilops tauschii), barley (Hordeum vulgare), rice (O. sativa), and purple false brome (Brachypodium distachyon) showed a divergence time of 35.3-40 MYA (Fig. 2d). The phylogenetic position of $D$. exilis as a basal taxon of the Paniceae allowed us to reconstruct the hypothetical ancestral genomic state of this tribe (Supplementary Data 2).

The hybridization of two genomes can result in genome instability, extensive gene loss, and reshuffling. As a consequence, one sub-genome may evolve dominancy over the other subgenome $24,31,33-35$. Using foxtail millet as a reference, the fonio A and B sub-genomes showed similar numbers of orthologous genes: 14,235 and 14,153, respectively. Out of these, 12,541 were retained as duplicates while 1694 and 1612 were specific to the A and B sub-genomes, respectively. The absence of sub-genome dominance was also supported by similar gene expression levels between homoeologous pairs of genes (Mann-Whitney $U$ test; $p$ =0.66; Supplementary Fig. 10 and Supplementary Data 3).

Evolutionary history of fonio and its wild relative. To get an overview of the diversity and evolution of fonio, we selected 166 D. exilis accessions originating from Guinea, Mali, Benin, Togo, Burkina Faso, Ghana, and Niger and 17 accessions of the proposed wild tetraploid fonio progenitor $D$. longiflora ${ }^{21}$ from Cameroon, Nigeria, Guinea, Chad, Sudan, Kenya, Gabon, and Congo for whole-genome re-sequencing (Supplementary Table 6). The selection was done from a collection of 641 georeferenced $D$. exilis accessions ${ }^{36}$ with the aim of maximizing diversity based on bioclimatic data and geographic origin. We obtained short-read sequences with an average of 45 -fold coverage for $D$. exilis (range 36-61-fold) and 20-fold coverage for D. longiflora (range 10-28-fold) (Supplementary Data 4). The average mapping rates of the raw reads to the CM05836 reference assembly were $85 \%$ and $68 \%$ for $D$. exilis and D. longiflora, respectively, with most accessions showing a mapping rate of $>80 \%$ (Supplementary Data 5). After filtering, 11,046,501 high quality bi-allelic single nucleotide polymorphisms (SNPs) were retained (Supplementary Table 7). Nine D. exilis and three D. longiflora accessions were discarded based on the amount of missing data. The error rate of variant calling (proportion of segregating sites in a re-sequenced CM05836 sample compared to the reference assembly) was $0.04 \%$, which is comparable to other studies $^{37}$. The re-sequenced CM05836 sample showed the lowest genetic divergence from the reference assembly of all resequenced accessions (Supplementary Fig. 11a). The SNPs were evenly distributed across the $18 \mathrm{D}$. exilis chromosomes, with a tendency toward a lower SNP density at the chromosome ends (Supplementary Fig. 11b). Approximately 30.2\% of the SNPs were gene-proximal (2 kb upstream or downstream of a coding sequence), $9.5 \%$ in introns and $6.2 \%$ in exons. Of the exonic SNPs, 354,854 (51.6\%) resulted in non-synonymous sequence changes, of which 6727 disrupted the coding sequence (premature 


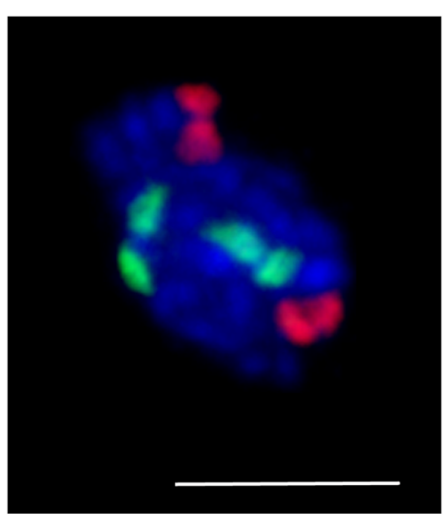

b
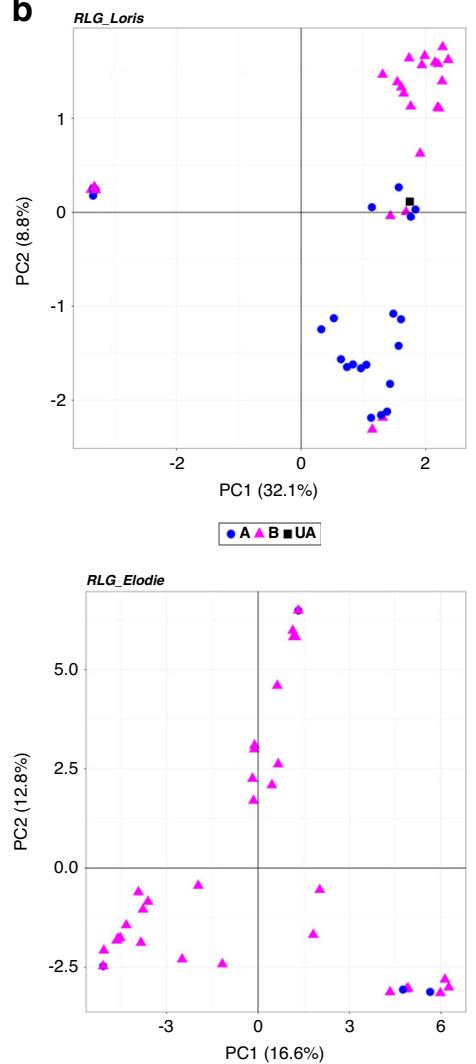

C

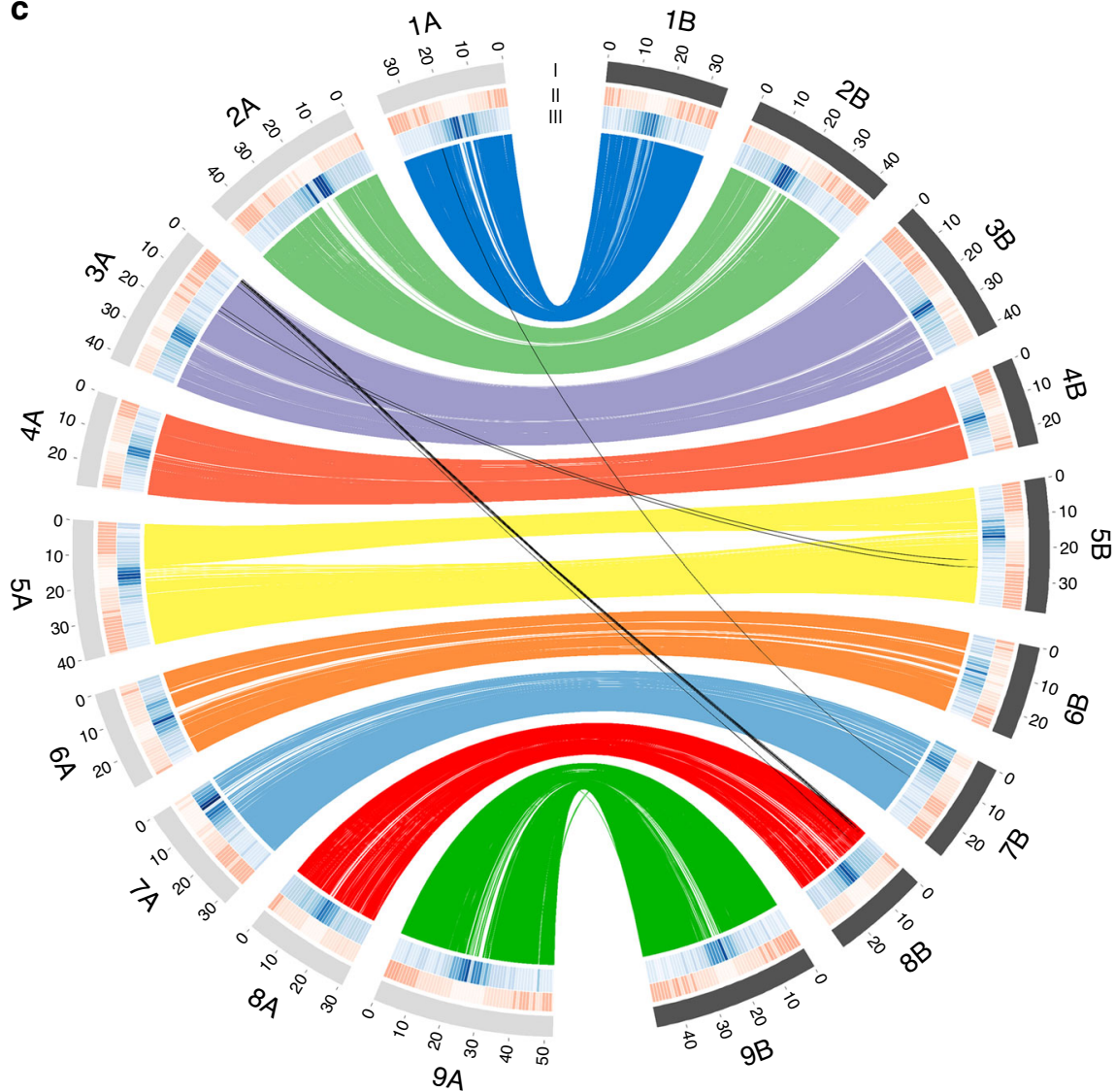

d

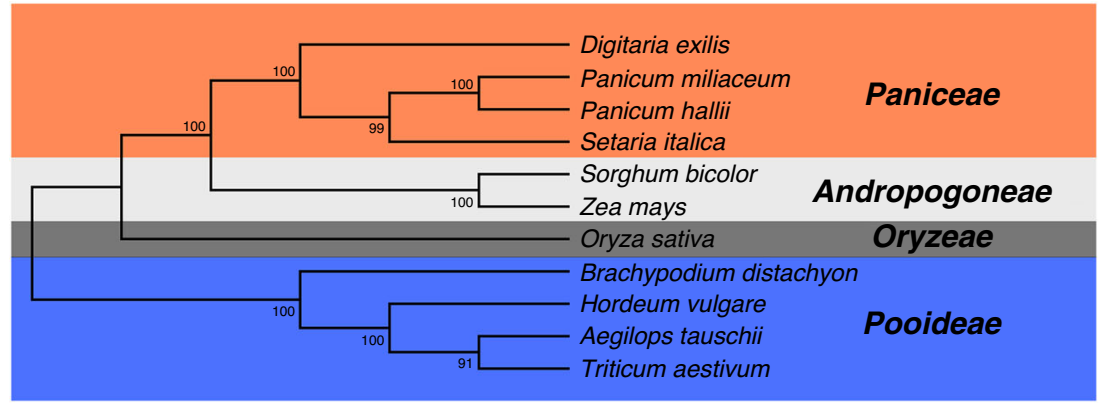

Fig. 2 Fonio genome features. a Representative example of oligo painting FISH on mitotic metaphase chromosomes. Shown are probes designed from pseudomolecules $9 \mathrm{~A}$ (green) and 9B (red) of the CM05836 assembly (scale bar $=5 \mu \mathrm{m}$ ). Oligo painting FISH experiment was repeated independently three times. b Principal component analysis (PCA) of the transposable element cluster RLG_Loris (upper panel) that allowed discrimination of the two subgenomes. Blue dots represent elements found on the $A$ sub-genome; pink triangles represent elements from the B sub-genome; black squares represent elements present on chromosome unanchored. PCA of the transposable element cluster RLG_Elodie (lower panel) that was specific to the B sub-genome. c Synteny and distribution of genome features. (I) Number and length of the pseudomolecules. The gray and black colors represent the two sub-genomes. (II, III) Density of genes and repeats along each pseudomolecule, respectively. Lines in the inner circle represent the homoeologous relationships. d Maximum likehood tree of 11 Poaceae species based on 30 orthologous gene groups. Topologies are supported by 1000 bootstrap replicates. Colors indicate the different clades.

stop codon). The remaining 333,296 (48.4\%) exonic SNPs represented synonymous variants. Forty-four percent of the total SNPs $(4,901,160$ SNPs) were rare variants with a minor allele frequency of $<0.01$ (Supplementary Fig. 12). The vast majority of the rare variants was found in a few very diverse $D$. longiflora accessions. The mean nucleotide diversity $(\pi)$ was $6.19 \times 10^{-4}$ and $3.68 \times 10^{-3}$ for $D$. exilis and $D$. longiflora, respectively. Genome-wide linkage disequilibrium (LD) analyses revealed a faster LD decay in D. longiflora $\left(r^{2} \sim 0.16\right.$ at $\left.70 \mathrm{~kb}\right)$ compared to D. exilis $\left(r^{2} \sim 0.20\right.$ at $\left.70 \mathrm{~kb}\right)$ (Supplementary Fig. 13).
A PCA showed a clear genetic differentiation between cultivated $D$. exilis and wild $D$. longiflora. The $D$. exilis accessions clustered closely together, while the $D$. longiflora accessions split into three groups (Fig. 3a). The D. longiflora group that showed the greatest genetic distance from $D$. exilis contained three accessions originating from Central (Cameroon) and East Africa (South Sudan and Kenya). The geographical projection of the first principal component (which separated wild accessions from the cultivated accessions) revealed that the $D$. exilis accessions genetically closest to 

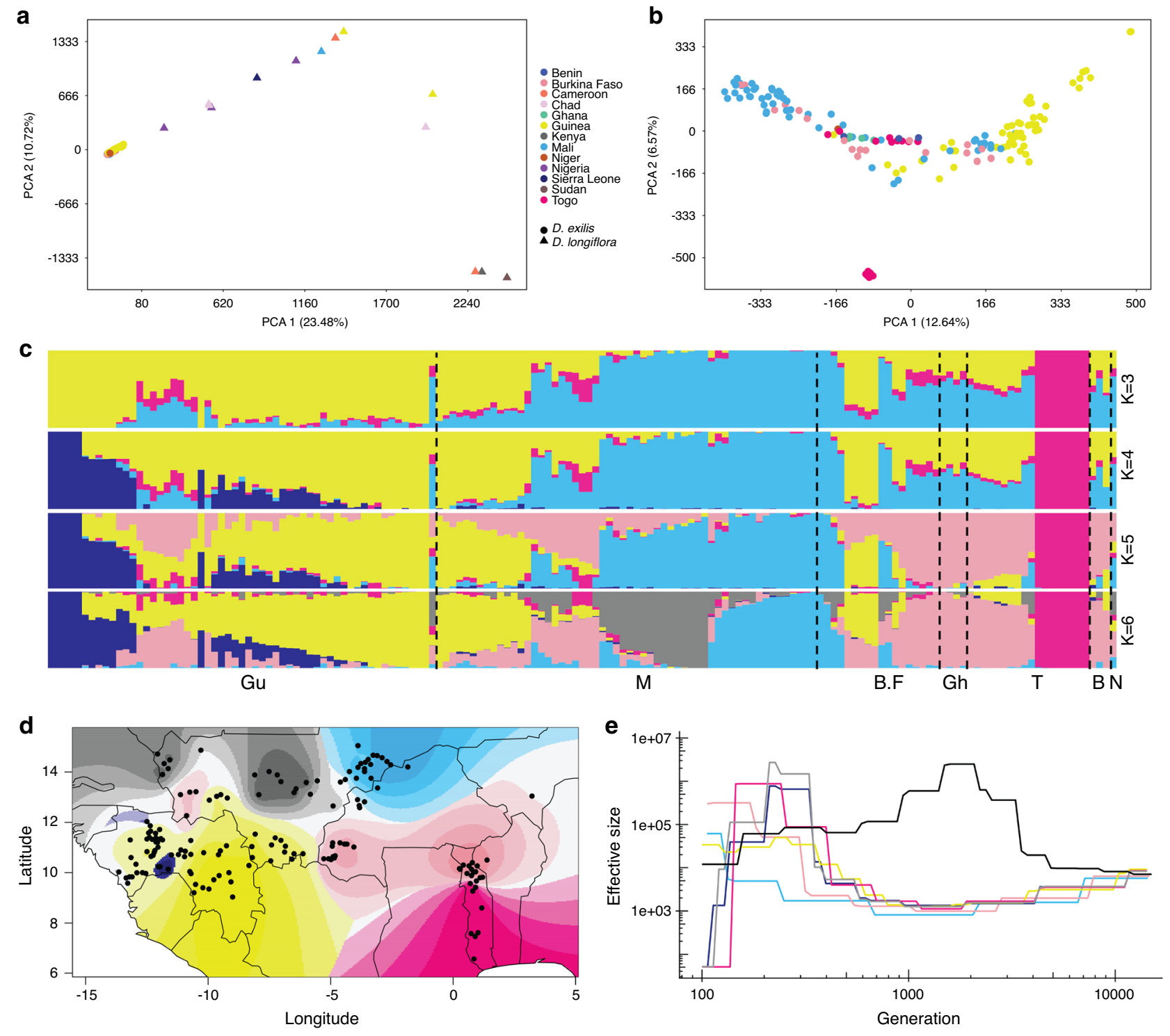

Fig. 3 Genetic diversity and structure of $\boldsymbol{D}$. exilis and $\boldsymbol{D}$. longiflora diversity panel. a Principal component analysis (PCA) of 157 D. exilis and 14 $D$. longiflora accessions using whole-genome single nucleotide polymorphisms (SNPs). D. exilis samples (circles), D. longiflora (triangles). b PCA of D. exilis accessions alone. Colors indicate the country of origin. $\mathbf{c}$ Population structure (from $K=3$ to $K=6$ ) of $D$. exilis accessions estimated with sNMF. Each bar represents an accession and the bars are filled by colors representing the likelihood of membership to each ancestry. Accessions are ordered from west to east; Guinea (Gu), Mali (M), Burkina Faso (B.F), Ghana (Gh), Togo (T), Benin (B), and Niger (N). d Geographic distribution of ancestry proportions of $D$. exilis accessions obtained from the structure analysis at $K=6$. The colors represent the maximal local contribution of an ancestry. Black dots represent the coordinates of $D$. exilis accessions. e Effective population size history of $D$. exilis groups based on $K=6$ and $D$. longiflora (in black).

D. longiflora originated from southern Togo and the west of Guinea (Supplementary Fig. 14a, b).

A PCA with $D$. exilis accessions alone revealed three main clusters. The second principal component separated eight accessions from southern Togo from the remaining accessions (Fig. 3b). In addition, five accessions from Guinea formed a distinct genetic group in the PCA. The remaining accessions were spread along the first axis of the PCA, mainly revealing a grouping by geographic location (Fig. 3b). The genetic clustering was confirmed by genetic structure analyses (Fig. 3c, d). The cross-validation error decreased with increasing $K$ and reached a plateau starting from $K=6$ (Supplementary Fig. 14c). At $K=3$, the eight South Togo accessions formed a distinct homogenous population. At $K=4$, the five accessions from Guinea were separated. With increasing $K$, the admixture plot provided some evidence that natural (climate and geography) and human (ethnicity and language) factors had an effect on shaping the genetic population structure of fonio (Fig. 3c, d, Supplementary Fig. 14d). We observed a significant correlation (Pearson's correlation; $p<0.05, \mathrm{df}=155)$ between the genetic population structure (first principal component of PCA) and climate (i.e., mean temperature and precipitation of the wettest quarters) as well as geography (i.e., latitude, longitude, and altitude) (Fig. 3b, Supplementary Fig. 15, Supplementary Data 6). The relationship between genetic differentiation (genetic distance matrix) and climate was still significant when accounting for geographic distance (partial Mantel test; Mantel $r=0.30 ; p=0.001$ ). A significant correlation was also observed between the genetic distance matrix and the dissimilarity matrices of ethnic and linguistic groups (fisher test; $p=0.0005$, Mantel test; Mantel $r$ ethnic $=-0.19 ;$ Mantel $r$ linguistic $=-0.13 ; p=0.001$ ). The effect of ethnic groups remained significant even when we 
controlled for geographic and climatic factors (ANCOVA; $p=$ 0.045; $\mathrm{df}=31$ ) (Supplementary Table 8). Association analyses based on climate variables revealed 38 and 179 loci that might be involved in adaptation to mean temperature and mean precipitation of the wettest quarters, respectively (Supplementary Fig. 16, Supplementary Data 7). Loci associated with temperature contained genes enriched for functions related to hormone metabolic processes, hormone biosynthesis, carbohydrate metabolic processes, homeostatic process, plant development processes, and plant growth (shoot apical meristem maintenance, root growth and development) (Supplementary Data 7). Similarly, genes associated with precipitation were enriched in functions related to plant development and growth (Supplementary Data 7). A genome-wide association study with ethnic groups revealed significant associations for 55 and 227 SNPs with Bambara and Fula ethnic groups, respectively (Supplementary Fig. 17, Supplementary Data 7). In particular, there were three prominent peaks on chromosomes $2 \mathrm{~A}, 2 \mathrm{~B}$, and $6 \mathrm{~A}$. The peaks on chromosomes $2 \mathrm{~A}$ and $2 \mathrm{~B}$ fell into a region that contained a homolog of the Arabidopsis WSD1 wax ester synthase/diacylglycerol acyltransferase gene ${ }^{38}$. WSD1 is involved in accumulation of waxes under drought stress, possibly indicating selection for adaptation to drought by certain ethnic groups. Unadmixed populations were mainly found at the geographic extremes of the fonio cultivation area in the north and south, whereas the accessions from the central regions of West Africa tended to show a higher degree of admixture (Supplementary Fig. 18a).
Plotting the spatial distribution of private SNPs (i.e., SNP present only once in a single accession) revealed a hotspot of rare alleles in Togo, Niger, the western part of Guinea, and southern Mali (Supplementary Fig. 18b, c). Rare allele diversity was lower in the eastern part of Guinea, and in southwest Mali. Inference of the $D$. exilis effective population size revealed a decline that started more than 10,000 years ago and reached a minimum between 2000 and 1000 years ago (Fig. 3e). Then, a steep increase of the effective population size occurred to a level that was $~ 100$ fold higher compared to the bottleneck.

Genomic footprints of selection and domestication. We used three complementary approaches to detect genomic regions under selection: (i) a composite likelihood-ratio (CLR) test based on site frequency spectrum (SFS) ${ }^{39}$, (ii) the nucleotide diversity $(\pi)$ ratios between $D$. exilis and $D$. longiflora over sliding genomic windows, and (iii) the genetic differentiation based on $F_{\mathrm{ST}}$ calculations, again computed over sliding windows. With the CLR test, 78 regions were identified as candidates for signatures of selection. The genetic diversity ratios and $F_{\mathrm{ST}}$ calculations revealed 311 and 208 regions, respectively (Fig. 4, Supplementary Data 8). We then searched for the presence of orthologs of known domestication genes in the regions under selection. The most striking candidate was one of the two orthologs of the rice grain size GS5 gene ${ }^{40}$ (Dexi3A01G0012320 referred to as DeGS5-3A) that was detected by genetic diversity ratio and $F_{\mathrm{ST}}$-based
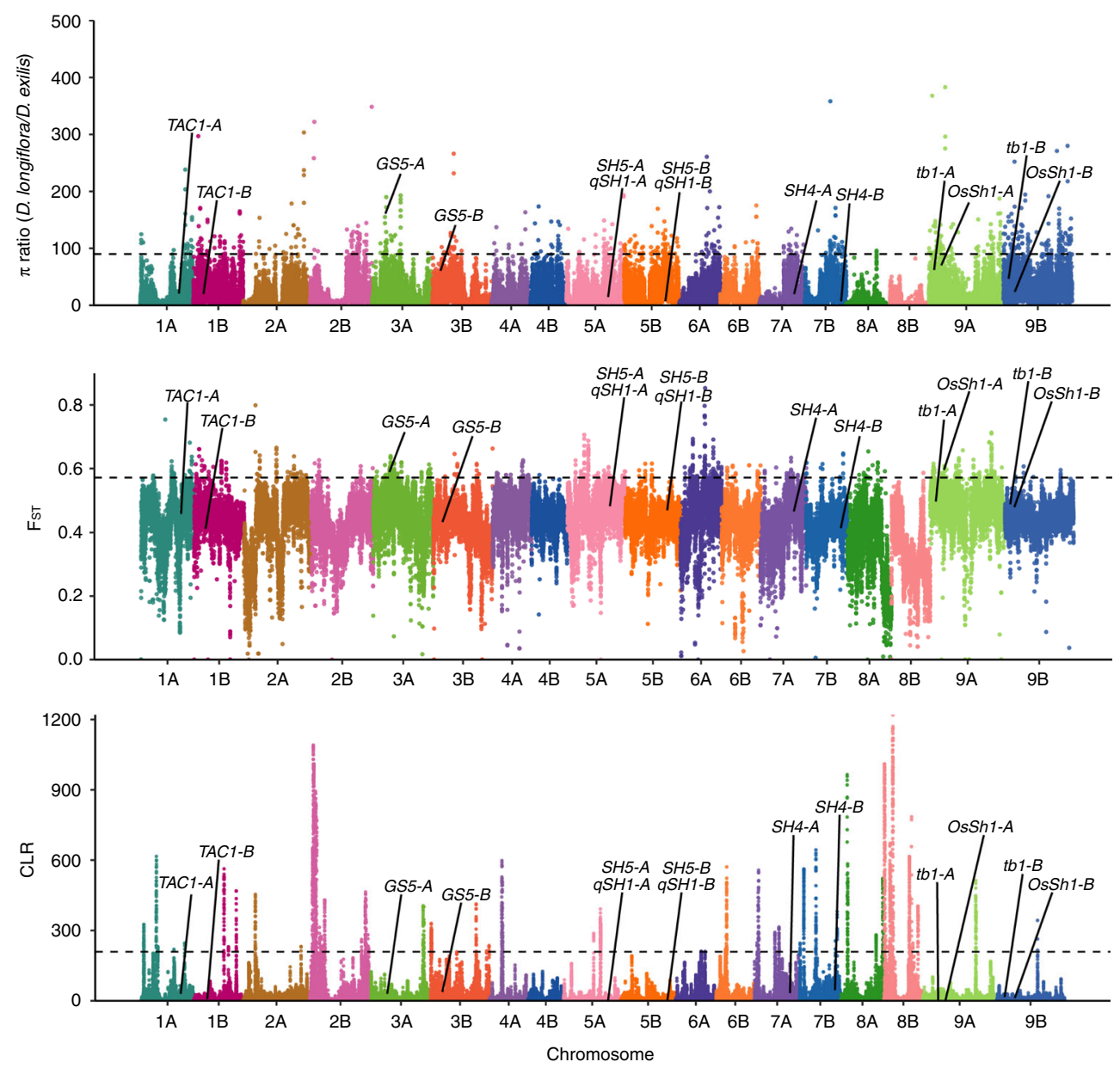

Fig. 4 Detection of selection in fonio. Manhattan plots showing detection of selection along the genome based on nucleotide diversity $(\pi)$ ratio, $F_{\mathrm{ST}}$ and SweeD (from top to bottom). The location of orthologous genes of major seed shattering and plant architecture genes are indicated in the Manhattan plots. The black dashed lines indicate the $1 \%$ threshold. Some extreme outliers in the $\pi$ ratio plot are not shown. 
a

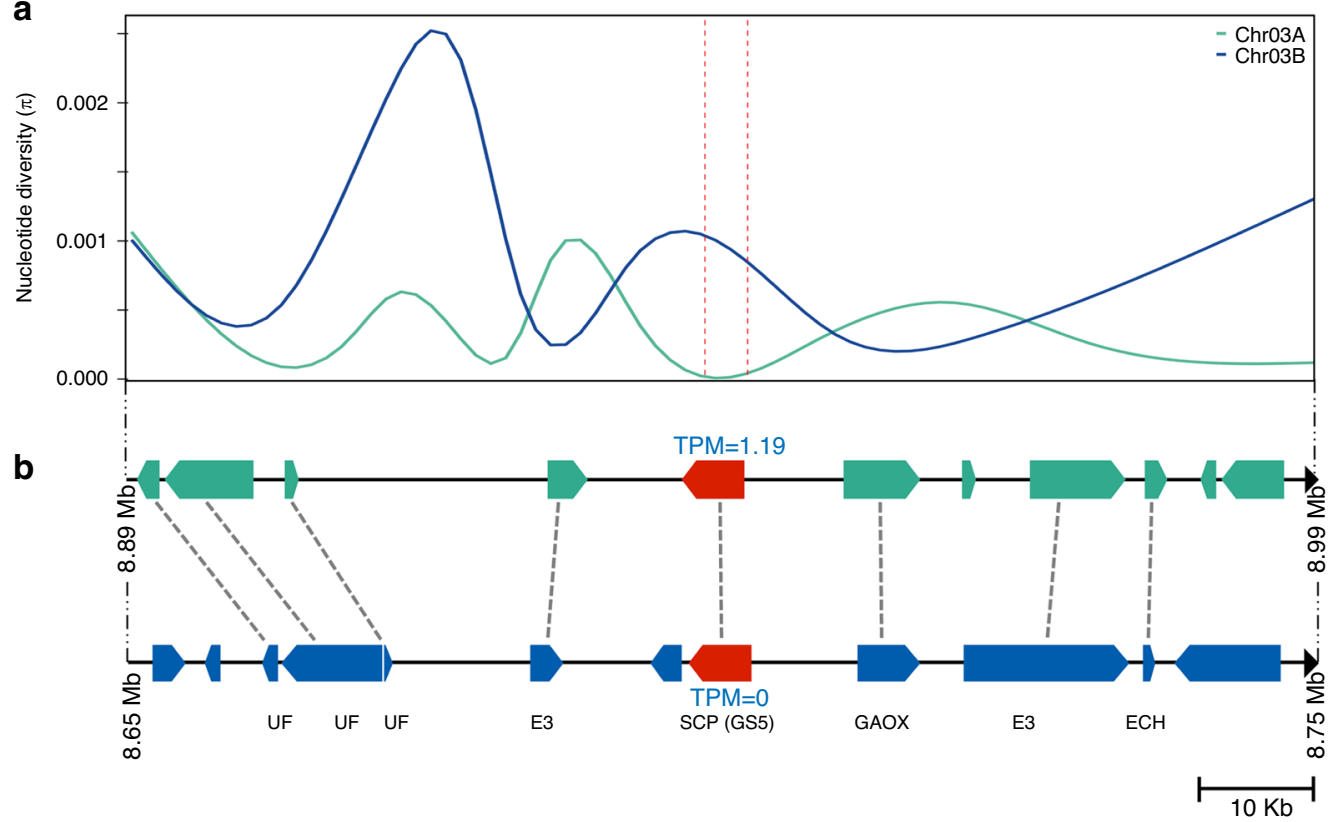

C

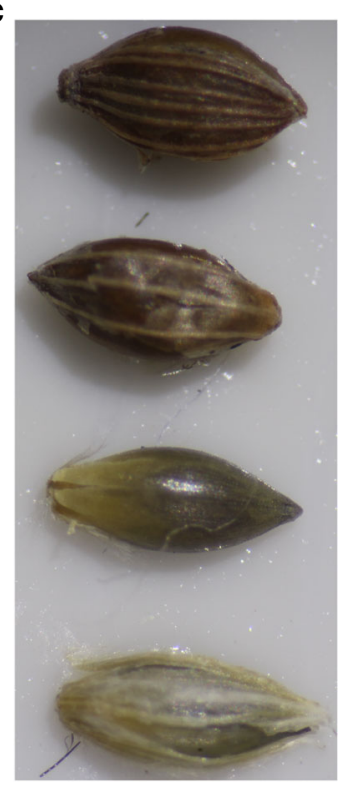

Fig. 5 Selective sweep at the GS5 locus in fonio. a Smoothed representation of nucleotide diversity $(\pi)$ in $D$. exilis in a $100 \mathrm{~kb}$ window surrounding the ortholog of the rice GS5 gene. The green curve shows $\pi$ around DeGS5-3A on chromosome 3A and the blue curve shows $\pi$ around DeGS5-3B on chromosome 3B. The dashed vertical red lines represent the location of the GS5 genes. The nucleotide diversity was calculated in overlapping $100 \mathrm{bp}$ windows every $25 \mathrm{bp}$. b Schematic representation of the annotated genes in the GS5 regions and the orthologous gene relationships between chromosomes 3A (green) and 3B (blue). UF protein of unknown function, E3 E3 ubiquitin ligase, SCP (GS5) serine carboxypeptidase (Dexi3A01G0012320), GAOX gibberellin 2-betadioxygenase, ECH golgi apparatus membrane protein. DeGS5-3A and DeGS5-3B are indicated in red. The numbers in blue above and below the GS5 orthologs show their respective expression in transcripts per million (TPM) in grain tissue. c Two grains of D. exilis (top) and D. longiflora (bottom).

calculations (Fig. 4). GS5 regulates grain width and weight in rice. D. exilis showed a dramatic loss of genetic diversity at the DeGS5$3 A$ gene (Fig. 5a, b). Domestication of fonio is associated with wider grains of $D$. exilis compared to grains of $D$. longiflora (Fig. 5c). The region of the GS5 ortholog on chromosome 3B (DeGS5-3B) was not identified as being under selection and showed higher levels of nucleotide diversity than the DeGS5-3A region (Fig. 5a, b). Only the DeGS5-3A but not the DeGS5-3B transcript was detected in the $D$. exilis RNA-Seq data from grains. This is in agreement with observations made in rice ${ }^{40}$, where a dominant mutation resulting in increased GS5 transcript levels affects grain size.

Another domestication gene that was detected in the selection scan was an ortholog of the sorghum Shattering 1 (Sh1) gene ${ }^{41}$. Shl encodes a YABBY transcription factor and the non-shattering phenotype in cultivated sorghum is associated with lower expression levels of $\operatorname{Sh} 1$ (mutations in regulatory regions or introns) or truncated transcripts. Domesticated African rice (O. glaberrima) carries a $45 \mathrm{~kb}$ deletion at the orthologous OsSh1 locus compared to its wild relative O. barthii ${ }^{42}$. Around $37 \%$ of the fonio accessions had a $60 \mathrm{~kb}$ deletion similar to O. glaberrima that eliminated the Sh1 ortholog on chromosome 9A (DeSh1-9A -Dexi9A01G0015055) (Fig. 6a). This deletion was identified by the lack of mapped reads and was confirmed by PCR amplification and Sanger sequencing. The homoeologous region including DeSh1-9B (Dexi9B01G0013485) on chromosome 9B was intact (Fig. 6b). Interestingly, accessions with the DeSh1-9A deletion showed a minor (7\%) but significant reduction in seed shattering (Fig. 6c) compared to accessions with the intact DeSh1$9 A$ gene (ANOVA; $p=0.008 ; \mathrm{df}=1$ ). Accessions carrying this deletion were distributed across the whole range of fonio cultivation, which suggests that the deletion is ancient and might have been selected for in certain regions.

\section{Discussion}

Here, we established a set of genomic resources that allowed us to comprehensively assess the genetic variation found in fonio, a cereal crop that holds great promises for agriculture in marginal environments. The analysis of fl-LTR-RT revealed two subgenome specific transposon clusters that experienced a peak of activity around 1.1-1.5 MYA, indicating that the two fonio subgenomes hybridized after this period ${ }^{43}$. The Ks analysis estimated that the two sub-genomes diverged prior to these transposon bursts-3 MYA, suggesting that fonio is an allotetraploid species.

The analysis of effective population size revealed a genetic bottleneck that was most likely associated with human cultivation and domestication. The large increase of effective size for fonio after a period of reduction was most probably due to the development and expansion of fonio cultivation. This expansion appears to be a recent event and occurred during the last millennium. The progression of the effective population size resembles the patterns observed for other domesticated crops, with a protracted period of cultivation followed by a marked bottleneck $^{44,45}$. In the case of fonio, this potential bottleneck appears to be milder compared to other crops. It has been observed that the effective population size of the wild rice $(O$. barthii) from West Africa followed a trend similar to the cultivated African rice (O. glaberrima), which has been interpreted as a result of environmental degradation ${ }^{44}$ rather than human selection. In contrast, no signal of population bottleneck was observed for the proposed wild fonio ancestor $D$. longiflora, indicating that the bottleneck observed in fonio is associated with human cultivation. We also highlighted the strong impact of geographic, climatic and anthropogenic factors on shaping the genetic diversity of fonio. Even if fonio is not a dominant crop across West Africa, it benefits from cultural embedding and plays a key role in ritual systems in many African cultures ${ }^{46}$. For 
a

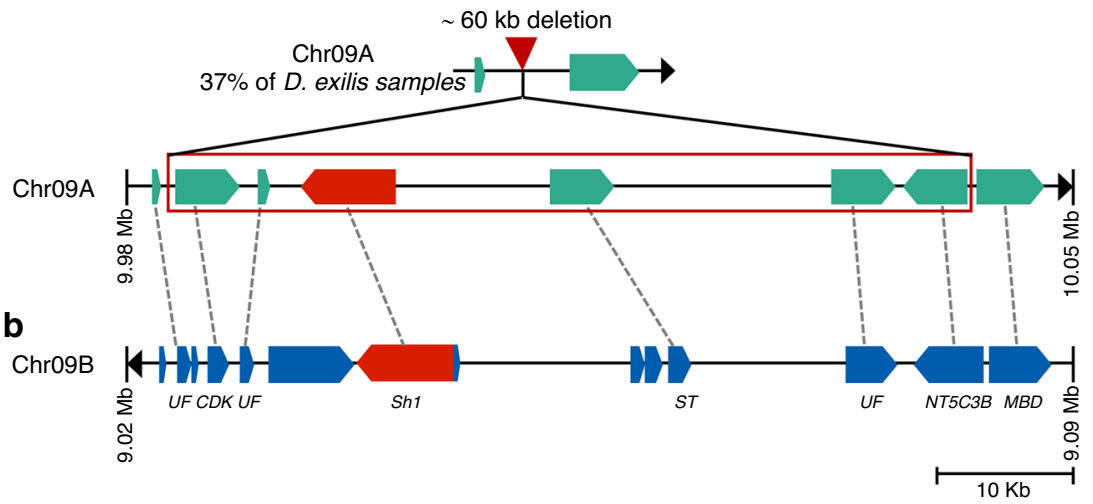

c

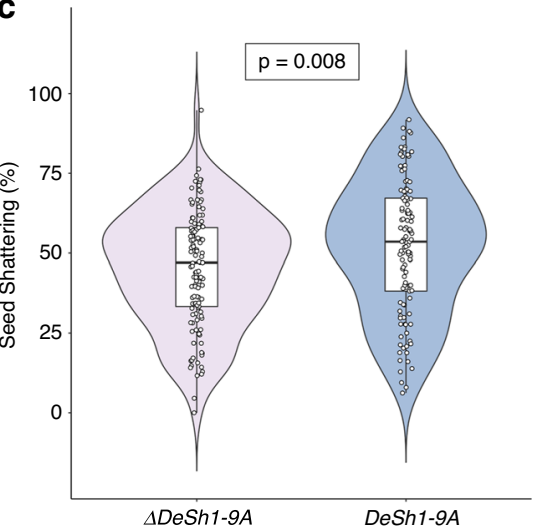

Fig. 6 Selective sweep at the Sh1 locus in fonio. a Schematic representation of genes in the orthologous regions of the sorghum Sh1 gene. The top most panel shows the region on fonio chromosome $9 \mathrm{~A}$ with the $60 \mathrm{~kb}$ deletion as it is found in $37 \%$ of all $D$. exilis accessions. The lower panel shows the genes in accessions without the deletion. The Sh1 ortholog DeSh1-9A is shown in red. $\mathbf{b}$ The Sh1 ortholog DeSh1-9B on chromosome 9B is present in all D. exilis accessions. The dashed lines represent orthologous relationships between the two sub-genomes. UF protein of unknown function, CDK cyclin-dependent kinase, Sh1 ortholog of sorghum Shattering 1 (Sh1), ST sulfate transporter; NT5C3B 7-methylguanosine phosphate-specific 5'-nucleotidase; MBD methylCpG-binding domain. c The violin plots show the probability distribution of the seed shattering percentage in fonio accessions carrying the DeSh1-9A deletion ( $\triangle$ DeSh1-9A) in pink compared to accessions with an intact DeSh1-9A in blue. The shape of the distribution indicates that the seed shattering percentages for both groups are concentrated around the median. The center of each plot depicts a boxplot, the box represents the interquartile range $(I Q R)$, the middle horizontal line represents the median, the vertical lines going down and up from the box are defined as first quartile ( -1.5 IQR) and third quartile ( $+1.5 \mathrm{IQR})$, respectively. Circles in the plot represent the seed shattering percentage calculated from individual fonio panicles, one circle per panicle, jitter option was added to show all individual observations that overlapped. Mean seed shattering was $45 \%$ in $\triangle D e S h 1-9 A$ ( $\pm 0.18 \mathrm{~s}$. d.) and $52 \%$ in DeSh1-9A ( \pm 0.22 s.d.). ( $n=$ Three individual panicles of $43 \Delta$ DeSh1-9A accessions and 39 DeSh-9A accessions, respectively; two-way ANOVA $p=0.008 ; \mathrm{df}=1)$.

example, we observed a striking genetic differentiation between D. exilis accessions collected from northern and southern Togo. This can be attributed to both climatic and cultural differences. While the southern regions of Togo receive high annual rainfalls, the northern parts receive $<1000 \mathrm{~mm}$ annual rainfall and there is a prolonged dry season ${ }^{47}$. Adoukonou-Sagbadja et al. ${ }^{47}$ also noted that there is no seed exchange between farmers of the two regions because of cultural factors.

Despite a genetic bottleneck and the reduction in genetic diversity, fonio still shows many 'wild' characteristics such as residual seed shattering, lack of apical dominance, and lodging. We show that orthologs of most of the well-characterized domestication genes from other cereals were not under strong selection in fonio. Interestingly, an ortholog of the rice GS5 gene (DeGS5-3A) was identified in a selective sweep. Dominant mutations in the GS5 promoter region were associated with higher GS5 transcript levels and wider and heavier grains in rice ${ }^{40}$. The DeGS5-3A gene showed a complete loss of diversity in the coding sequence in $D$. exilis, suggesting a strong artificial selection for larger grains. In contrast, DeSh1-9A showed evidence for a partial selective sweep ${ }^{48}$. The nonshattering phenotype associated with the Shl locus in sorghum is recessive $^{41}$ and the deletion of a single DeSh 1 copy only resulted in a quantitative reduction of shattering that might have been selected for in some but not all regions. Whether the $60 \mathrm{~kb}$ deletion including DeSh1-9A represents standing genetic variation or arose after fonio domestication cannot be determined. The deletion was not identified in any of the 14 re-sequenced $D$. longiflora accessions. Targeting the DeSh1-9B locus on chromosome 9B in accessions that carry the DeSh1-9A deletion through mutagenesis or genome editing could produce a fonio cultivar with significantly reduced seed shattering, which would form a first important step towards a significant improvement of this crop.

\section{Methods}

Plant material. The plant material used in this study comprised a collection of 641 D. exilis accessions maintained at the French National Research Institute for
Development (IRD), Montpellier, France ${ }^{36,49}$. The collection was established from 1977 to 1988. For genome re-sequencing, a panel of 166 accessions (Supplementary Data 6) was selected based on geographic and bioclimatic data. One plant per accession was chosen for DNA extraction and sequencing. Inflorescences of sequenced $D$. exilis plants were covered with bags to prevent outcrossing. Grains of sequenced plants were collected and kept for further analyses. In addition, 17 accessions of $D$. longiflora were collected from specimens stored at the National Museum of Natural History of Paris (Paris, France), IFAN Ch. A. Diop (Dakar, Senegal), National Herbarium of The Netherlands (Leiden, Netherlands), and Cirad (Montpellier, France) (Supplementary Table 6).

Flow cytometry for genome size estimation. The amount of nuclear DNA in fonio was measured by flow-cytometry ${ }^{50}$. In brief, six different individual plants representing accession CM05836 were measured three times on three different days using a CyFlow ${ }^{\circledast}$ Space flow cytometer (Sysmex Partec GmbH, Görlitz, Germany) equipped with a $532 \mathrm{~nm}$ green laser. Soybean (Glycine max cultivar Polanka; 2C = $2.5 \mathrm{pg}$ ) was used as an internal reference standard. The gain of the instrument was adjusted so that the peak representing G1 nuclei of the standard was positioned approximately on channel 100 on a histogram of relative fluorescence intensity when using a 512-channel scale. The low level threshold was set to channel 20 to eliminate particles with the lowest fluorescent intensity from the histogram. All remaining fluorescent events were recorded with no further gating used. 2C DNA contents (in pg) were calculated from the mean G1 peak (interphase nuclei in G1 phase) positions by applying the formula:

$2 \mathrm{C}$ nuclear DNA content $=\frac{(\text { sample G1 peak mean }) \times(\text { standard 2C DNA content })}{(\text { standard G1 peak mean })}$.

DNA contents in pg were converted to genome sizes in bp using the conversion factor $1 \mathrm{pg} \mathrm{DNA}=0.978 \mathrm{~Gb}^{51}$.

Reference genome sequencing and assembly. High molecular weight (HMW) genomic DNA was isolated from a single CM05836 plant with a dark-treatment of $48 \mathrm{~h}$ before harvesting tissue from young leaves. Two paired-end and three matepair libraries were constructed with different insert sizes ranging from $450 \mathrm{bp}$ to $10 \mathrm{~kb}$. The $450 \mathrm{bp}$ paired-end library was sequenced on an Illumina Hi-Seq 2500 instrument. The other libraries were sequenced on a Illumina NovaSeq 6000 instrument. In addition, one $10 \times$ linked read library was constructed and sequenced with an Illumina NovaSeq 6000 instrument, yielding 75-fold coverage. A de novo whole genome assembly (WGA) was performed with the DeNovoMAGIC3 software (NRGene).

For the super-scaffolding, one Hi-C library was generated using the Dovetail ${ }^{\mathrm{TM}}$ Hi-C Library Preparation Kit and sequenced on one lane of an Illumina HiSeq 
4000 instrument, yielding 128-fold coverage. The assembly to Hi-C superscaffolds was performed with the HiRise ${ }^{\mathrm{TM}}$ software v2.0. This generated 18 large super-scaffolds with a size between 25.5 and $49.1 \mathrm{Mb}$ (total size $=642.8 \mathrm{Mb}$ with $\mathrm{N} 50=39.9 \mathrm{Mb}$ ), while the 19 th longest super-scaffold was only $1.5 \mathrm{Mb}$ in size, indicating that the 18 longest super-scaffolds correspond to the $18 \mathrm{D}$. exilis chromosomes.

To generate a BioNano optical map, ultra HMW DNA was isolated using the QIAGEN Genomic-tip 500/G kit (Cat no./ID: 10262) from plants grown in a greenhouse with a dark treatment applied during $48 \mathrm{~h}$ prior to collecting leaf tissue. Labeling and staining of the HMW DNA were performed according to the Bionano Prep Direct Label and Stain (DLS) protocol (30206-Bionano Genomics) and then loaded on one Saphyr chip. The optical map was generated using the Bionano Genomics Saphyr System according the Saphyr System User Guide (30247Bionano Genomics). A total of $1114.6 \mathrm{~Gb}$ of data were generated, but only $210.6 \mathrm{~Gb}$ of data corresponding to molecules with a size larger than $150 \mathrm{~kb}$ were retained. Hybrid scaffolding between the WGA and the optical maps was done with the hybridScaffold pipeline (with the Bionano Access v1.4 default parameters). In total, 39 hybrid scaffolds ranging from $550 \mathrm{~kb}$ to $40.9 \mathrm{Mb}$ (total length $657.3 \mathrm{Mb}$ with $\mathrm{N} 50=22.6 \mathrm{Mb}$ ) were obtained (Supplementary Table 3 )

To construct the final chromosome-scale assembly, we integrated the Hi-C super-scaffolds and the hybrid scaffold manually. The 39 hybrid scaffolds generated with the optical map were aligned to the $18 \mathrm{Hi}-\mathrm{C}$ super-scaffolds produced by HiRise (Supplementary Table 9). The hybrid scaffolds detected two chimeric DeNovoMAGIC3 scafflods that most likely arose from wrong connections in the putative centromeres. The final chromosome-level assembly was constructed as follows: (i) We used the hybrid assembly to break the two chimeric DeNovoMAGIC3 scaffolds, (ii) merged and oriented the hybrid scaffolds using the Hi-C super scaffolds, and (iii) merged the remaining contigs and scaffolds into an unanchored chromosome.

A second genome assembly was produced using the TRITEX pipeline ${ }^{27}$. Briefly, the initial step using Illumina sequencing data (pre-processing of paired-end and mate-pair reads, unitig assembly, scaffolding and gap closing) was done according the instructions of the TRITEX pipeline [https://tritexassembly.bitbucket.io/]. Integration of $10 \times$ and $\mathrm{Hi}-\mathrm{C}$ reads was done differently. For the $10 \times$ scaffolding, we used tigmint vl.1.252 to detect and cut sequences at positions with few spanning molecules, arks v1.0.3 $3^{53}$ to generate graphs of scaffolds with connection evidence, and LINKS $^{54}$ for a second step of scaffolding. For the Hi-C data, we used BWA ${ }^{55}$ to map the reads against the previous scaffolds and juicer tools v $1.5^{56}$ for the superscaffolding.

Preparation of FISH probes and cytogenetic analyses. Libraries of $45 \mathrm{bp}$ long oligomers specific for each fonio pseudomolecule were designed using the Chorus software v1.126 [https://github.com/forrestzhang/Chorus] with the following criteria: -p CGTGGTCGCGTCTCA -1 45 -homology 75 -d 10. The number of oligomers per pseudomolecule was adjusted to ensure uniform fluorescent signals along the entire chromosomes after fluorescence in situ hybridization (FISH). In total, 310,484 oligomers were selected (between 12,647 and 20,000 oligomers per library) and were synthesized by Arbor Biosciences (Ann Arbor, MI, USA). To prepare chromosome painting probes for FISH, the oligomer libraries were labeled directly using 6-FAM or CY3, or indirectly using digoxigenin or biotin. A tandem repeat CL10 with a 314 bp long repetitive unit (155 bp long subunit) was identified after the analysis of fonio DNA repeats with the RepeatExplorer software ${ }^{57}$. A 20 bp oligomer was designed based on the CL10 sequence using the Primer3 software ${ }^{58}$ labeled by CY 3 and used for FISH.

Chromosome spreads were prepared from actively growing roots $(\sim 1 \mathrm{~cm} \text { long })^{59}$. Roots were collected into $50 \mathrm{mM}$ phosphate buffer ( $\mathrm{pH} 7.0$ ) containing $0.2 \% \beta$ mercaptoethanol, pre-treated in $0.05 \%$ 8-hydroxyquinoline for three hours at room temperature, fixed in 3:1 ethanol:acetic acid fixative overnight and stored in $70 \%$ ethanol at $-20^{\circ} \mathrm{C}$. Chromosome spreads prepared from $\sim 20$ roots were washed in distilled water, $1 \times \mathrm{KCl}$ buffer $(\mathrm{pH} 4)$ and root tips were incubated in enzyme mixture containing $4 \%$ cellulase and $2 \%$ macerozyme for $56 \mathrm{~min}$ at $37^{\circ} \mathrm{C}$. The reaction was stopped with TE buffer ( $\mathrm{pH} 7.6)$, root tips were washed in $100 \%$ ethanol, then $30 \mu \mathrm{l}$ of 9:1 ice-cold acetic acid:methanol mixture was added and root tips were broken with tweezers and dropped onto slide placed in a humid box. The preparations were air-dried, postfixed in $4 \%(\mathrm{v} / \mathrm{v})$ formaldehyde solution in $2 \times \mathrm{SSC}$ solution and used for fluorescence in situ hybridization (FISH). FISH was performed with the chromosome painting probes and a probe for the CL10 tandem repeat using a hybridization mixture $(30 \mu \mathrm{l})$ containing $50 \%$ formamide, $10 \%$ dextran sulfate in $2 \times$ SSC and $10 \mathrm{ng} / \mu \mathrm{l}$ of labeled probe. Hybridization was carried out overnight at $37^{\circ} \mathrm{C}$. Digoxigenin-labeled and biotin-labeled probes were detected using anti-digoxigenin-FITC (Roche Applied Science) and streptavidin-Cy3 (ThermoFisher Scientific/Invitrogen), respectively. The preparations were mounted in Vectashield with DAPI (Vector laboratories, Ltd., Peterborough, UK) to counterstain the chromosomes and the slides were examined with an Axio Imager Z.2 Zeiss microscope (Zeiss, Oberkochen, Germany) equipped with a Cool Cube 1 camera (Metasystems, Altlussheim, Germany) and appropriate optical filters. The capture of fluorescence signals and measure of chromosome lengths were done using the ISIS software 5.4.7 (Metasystems) and final image adjustment was done in Adobe Photoshop CS5.
Analysis of fl-LTR-RT. fl-LTR-RT were identified using both LTRharvest ${ }^{60}$ (-minlenltr 100 -maxlenltr 40000 -mintsd 4 -maxtsd 6 -motif TGCA -motifmis 1 -similar 85 -vic 10 -seed 20 -seqids yes) and LTR_finder ${ }^{61}$ (-D 40000 -d 100 -L 9000 -1 50 -p 20 -C -M 0.9). Then, the candidate LTRs were filtered using LTR_retriever ${ }^{62}$.

fl-LTRs were classified into families using a clustering approach using MeShClust $2^{63}$ with default parameters and manually curated with dotter ${ }^{64}$ to discard wrongly assigned LTRs. Only LTR-RT families with at least 30 intact copies were considered in genetics analyses, where different families represented 'populations' and each fl-LTR-RT copy of a family was considered as an individual. A multiple sequence alignment was performed within each family using Clustal $\mathrm{Omega}^{65}$ and the polymorphic sites were identified and converted into variant call format (VCF) using msa2vcf.jar [https://github.com/lindenb/jvarkit]. A principal component analysis (PCA) was performed for each fl-LTR-RT family using the R packages vcfR v.1.8.0 $0^{66}$ and adegenet v2.1.1 ${ }^{67}$. Results were visualized with ggplot2 v3.3.268.

The approximate insertion dates of the fl-LTR-RTs were calculated using the evolutionary distance between two LTR sequences with the formula $T=K / 2 \mu$, where $K$ is the divergence rate approximated by percent identity and $\mu$ is the neutral mutation rate. The mutation rate used was $\mu=1.3 \times 10^{-8}$ mutations per bp per year ${ }^{69}$.

Gene annotation. A combination of homology-based and de novo approaches was used for repeat annotation using the RepeatModeler software [http://www. repeatmasker.org/RepeatModeler/] and the RepeatExplorer software ${ }^{57}$. The results of RepeatModeler, RepeatExplorer and LTR_retriever were merged into a comprehensive de novo repeat library using USEARCH v.1 $17^{70}$ with default parameters (-id 0.9). The final repeats were classified using the RepeatClassifier module with the NCBI engine and then used to mask the CM05836 assembly.

The protein coding genes were predicted on the masked genome using MAKER $(\mathrm{v} 3.01 .02)^{71}$. Transcriptome reads from flag leaf, grain, panicle and above-ground seedlings were filtered for ribosomal RNA using SortMeRNA v2.1 ${ }^{72}$ and for adaptor sequences, quality and length using trimmomatic $\mathrm{v} 0.38^{73}$. Filtered reads were mapped to the reference sequence using STAR v.2.7.0 $\mathrm{d}^{74}$ and the alignments were assembled with StringTie v.1.3.5 $5^{75}$ to further be used as transcript evidence. Protein sequences from Arabidopsis thaliana ${ }^{76}$, S. italica ${ }^{28}$, S. bicolor ${ }^{77}$, Z. mays $^{78}$ and O. sativa ${ }^{79}$ were compared with BLASTX ${ }^{80}$ to the masked pseudomolecules of CM05836 and alignments were filtered with Exonerate v.2.2.0 $0^{81}$ to search for the accurately spliced alignments. A de novo prediction was performed using ab initio softwares GeneMark-ES v.3.54 ${ }^{82}$, SNAP v.2006-07-28 ${ }^{83}$, and Augustus v.2.5.5 $5^{84}$ Three successive iterations of the pipeline MAKER were performed in order to improve the inference of the gene models and to integrate the final consensus genes. Finally, the putative gene functions were assigned using the UniProt/ SwissProt database (Release 2019_08 - [https://www.uniprot.org/]).

Synteny and comparative genome analysis. To identify syntenic relationships, BLASTP $\left(E\right.$-value $1 \times 10^{-5}$ ) was first used to perform all-versus-all protein comparisons between CM05836 and S. italica ${ }^{28}$, Panicum miliaceum ${ }^{35}$, Panicum hal-

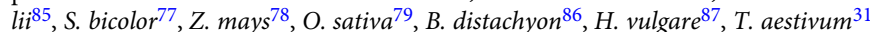
and A. tauschii ${ }^{88}$. Then, MCScanX ${ }^{89}(-\mathrm{m} 25 ;-\mathrm{s} 10)$ was used for pairwise synteny block detection. For all the comparisons, fonio sub-genomes A and B were analyzed independently and only 1:1 relationships were retained (1:2 relationships for tetraploid broomcorn millet and the ancient tetraploid maize, where individual sub-genomes are not clearly phased).

To estimate the divergence time, we calculated the synonymous substitution rates $(K s)$ for each homoeologous and orthologous pair. Briefly, nucleotide sequences were aligned with clustalW $\mathrm{W}^{90}$ and the $K$ s were calculated using the CODEML program in PAML ${ }^{91}$. Then, time of divergence events was calculated using $T=K \mathrm{~s} / 2 \lambda$, based on the clock- $(\lambda)$ estimated for grasses of $6.5 \times 10^{-9} 92$. We used the maximum likelihood for gene-order analysis (MLGO) tool ${ }^{93}$ to reconstruct the hypothetical ancestral genome of the Paniceae tribe from syntenic-block data of D. exilis, S. italica, P. miliaceum, and P. hallii. Syntenic blocks were identified using MCScanX ${ }^{89}$ using the B sub-genome of $D$. exilis as reference. Then, individual syntenic blocks across all selected species were analyzed according their physical position and orientation in order to combine them into syntenic-block markers ${ }^{94}$ (Supplementary Data 2). Syntenic-block markers along with the known species tree were provided as inputs to the MLGO server to infer the ancestral state of the Paniceae.

To analyze genome dominance, the quantification of transcript abundance and expression were calculated using RSEM v1.3.195 and genes were considered as expressed when expression was $>0.5$ TPM in at least one tissue. To study the homoeolog expression bias a two-sided Mann-Whitney $U$ test was used to examine if the TPM values of sub-genomes A and B differed significantly.

Re-sequencing of $\boldsymbol{D}$. exilis and $\boldsymbol{D}$. longiflora accessions. One or two young leaves from $D$. exilis or $D$. longiflora seedlings were collected in $2 \mathrm{ml}$ Eppendorf tubes, flash-frozen in liquid nitrogen, and ground with glass beads in a SPEX SamplePrep Geno/Grinder 2010. Samples were mixed with $1.2 \mathrm{ml} 2 \times$ CTAB buffer (2\% CTAB, $200 \mathrm{mM}$ Tris/ $\mathrm{HCl}$ (pH 8), $20 \mathrm{mM}$ EDTA, $1.4 \mathrm{M} \mathrm{NaCl}, 1.0 \%$ PVP, $0.28 \mathrm{M} \beta$ - 
mercaptoethanol) and incubated at $65^{\circ} \mathrm{C}$ with periodic mixing for $60 \mathrm{~min}$. Samples were cooled to room temperature and centrifuged at $2000 \times g$ for $10 \mathrm{~min}$. Subsequently, $900 \mu \mathrm{l}$ of the supernatant was transferred to a fresh $2 \mathrm{ml}$ Eppendorf tube and incubated with $800 \mu$ ldichloromethane:isoamyl alcohol (24:1) in an overheadshaker at half-speed at $4{ }^{\circ} \mathrm{C}$ for $15 \mathrm{~min}$. After centrifugation at $10,000 \times g$ for $15 \mathrm{~min}, 800 \mu \mathrm{l}$ of the supernatant was transferred to a fresh $2 \mathrm{ml}$ Eppendorf tube and incubated with $5 \mu \mathrm{l}$ RNase A (10 mg/ml, EN0531, ThermoFisher Scientific) at $37^{\circ} \mathrm{C}$ for $15 \mathrm{~min}$. DNA was precipitated by adding $560 \mu \mathrm{l}$ isopropanol and mixing the tubes by inversion. Precipitated DNA was pelleted by centrifugation at $4{ }^{\circ} \mathrm{C}$ and $10,000 \times g$ for $10 \mathrm{~min}$ and the supernatant discarded. The DNA pellet was washed in a first ethanol wash (76\% ethanol, $200 \mathrm{mM}$ sodium acetate) for $15 \mathrm{~min}$ and in a second ethanol wash (76\% ethanol, $10 \mathrm{mM}$ ammonium acetate) for $5 \mathrm{~min}$. Subsequently, DNA pellets were air-dried to remove residual ethanol and eluted in 50 $\mu \mathrm{l}$ TE buffer (10 mM Tris/HCl (pH 8), $1 \mathrm{mM}$ EDTA). Extracted DNA was quantified with the Qubit dsDNA HS Assay (Q32851, ThermoFisher Scientific), the purity was confirmed by checking $260 / 280$ and $260 / 230$ ratios on a Nanodrop spectrophotometer, and the integrity was confirmed by analyzing $1 \mu \mathrm{l}$ per sample on a $1 \%$ TAE agarose gel. Library preparation and sequencing were performed by Novogene. Briefly, $1.0 \mu \mathrm{g}$ DNA per sample was used as input material for the DNA sample preparations. Sequencing libraries were generated using the NEBNext $^{\circledR}$ Ultra II DNA Library Prep Kit following manufacturer's instructions. Libraries were sequenced using an Illumina NovaSeq 6000 system.

Mapping of re-sequencing data and variant calling. For quality control of each sample, raw sequence reads were analyzed with the fastqQC tool-v0.11.7 and lowquality reads were filtered with trimmomatic-v0.38 ${ }^{73}$ using the following criteria: LEADING:20; TRAILING:20; SLIDINGWINDOW:5:20 and MINLEN:50. The filtered paired-end reads were then aligned for each sample individually against the CM05836 reference assembly using BWA-MEM (v0.7.17-r1188) ${ }^{55}$ followed by sorting and indexing using samtools (v1.6) ${ }^{96}$. Alignment summary, base quality score and insert size metrics were collected and duplicated reads were marked and read groups were assigned using the Picard tools [http://broadinstitute.github.io/ picard/]. Variants were identified with GATK v3.8 ${ }^{97}$ using the emitRefConfidence function of the HaplotypeCaller algorithm to call SNPs and InDels for each accession followed by a joint genotyping step performed by GenotypeGVCFs. To obtain high confidence variants, we excluded SNPs and InDels with the VariantFiltration function of GATK with the criteria: QD $<2.0$; FS $>60.0$; MQ $<40.0$; MQRankSum $<-12.5$; ReadPosRankSum $<-8.0$ and SOR $>4.0$. The complete automated pipeline has been compiled and is available on github [https://github. com/IBEXCluster/IBEX-SNPcaller].

A total of 36.5 million variants were called. Raw variants were filtered using GATK v3.8 ${ }^{97,98}$ and VCFtools v0.1.17 ${ }^{99}$. Variants located on chromosome unanchored, InDels, 'SNP clusters' defined as three or more SNPs located within $10 \mathrm{bp}$, missing data $>10 \%$, low and high average SNP depth $(14 \leq \mathrm{DP} \geq 42)$, and accessions having more than $33 \%$ of missing data were discarded. Only biallelic SNPs were retained to perform further analyses representing a final VCF file of $11,046,501$ SNPs (Supplementary Table 7). These variants were annotated using snpEff $\mathrm{v} 4.3^{100}$ with the CM05836 gene models.

Genetic diversity and population structure. Genetic diversity and population structure analyses were performed using $D$. exilis and D. longiflora accessions together, or with $D$. exilis accessions alone. PCA and individual ancestry coefficients estimation were performed using the R package LEA v2.0 ${ }^{101}$. The geographical projection of the first PCA axis of the $D$. exilis samples was done and visualized using the Kriging function in the fields v10.3 R package [https://cran.rproject.org/web/packages/fields] ${ }^{102}$. For ancestry coefficients analyses, the snmf function was used with 10 independent runs for each $K$ from $K=2$ to $K=10$. The optimal $K$, indicating the most likely number of ancestral populations, was determined with the cross-validation error rate. For the two analyses, we considered only SNPs present in at least two accessions (i.e., private SNPs were excluded). We also studied the geographic distribution of private $\mathrm{SNPs}^{103}$ (i.e., SNP present only once in a single accession). Genome-wide pairwise LD was estimated independently for D. exilis (2,617,322 SNPs) and D. longiflora (9,839,152 SNPs). LD decay $\left(r^{2}\right)$ was calculated using the tool PopLDdecay v $3.40^{104}$ in a $500 \mathrm{~kb}$ distance and plotted using the ggplot2 v3.3.2 package in $\mathrm{R}^{68}$.

\section{Association of climate, geography, and social factors with the population} genetics structure. Natural (i.e., climate, geography) and human (i.e., ethnic and linguistic) factors were tested for an association with the genetic structure of $D$. exilis accessions. Bioclimate data and ethnic and linguistic data were extracted for each of the 166 re-sequenced D. exilis accessions from WorldClim v1.4 [https:// www.worldclim.org/data/v1.4/worldclim14.html] ${ }^{105}$, passport data (ethnic groups), and Ethnologue v16 (language, [https://www.ethnologue.com/]). The associations between the genetic structure and different factors were evaluated statistically using Pearson's correlation coefficient test (two-sided) for the bioclimatic and geographic data with the $\mathrm{R}$ package stats v3.6.0 (function cor). For the associations of social factors, two-sided Mantel tests (ecodist R package v2.0.5 [https://rdrr.io/cran/ ecodist/]) with 999 permutations were performed between the genetic distance matrix of $D$. exilis and the dissimilarity matrix of social factors as inputs. Fisher's exact tests (stats R package v3.6.0) were performed with the structure data at $K=6$ and accessions having ancestry thresholds of $>70 \%$ as input. The $p$-values were computed by Monte Carlo simulation. We used analysis of covariance (ANCOVA) to test for the impact of ethnolinguistic factors on the first PCA coordinates while controlling for other covariates: climatic and geographic variables.

Synthetic climate variables were retrieved from the WorldClim v1.4 database ${ }^{105}$ We focused our analysis on two climate variables that were the most pertinent to annual species, i.e., mean temperature of the wettest quarter (bio8) and mean precipitation of the wettest quarter (bio 16), respectively. A GWAS with these climate variables has been performed ${ }^{106}$. Briefly, we first used the impute method from the R package LEA v2.0 $0^{101}$ to fill the gaps in the genotypic matrix in order to achieve greater power. We then filtered out SNPs with a MAF below $5 \%$. We used three different models of association to perform the GWAS, efficient mixed-model association (EMMA) ${ }^{107}$ using the R package emma v1.1.2, mixed linear model (MLM) as implemented in the R package gapit v3.0 108 and latent factor mixed models (LFMM) using LFMM2 ${ }^{109}$ as implemented in lfmm R package v2.0. For gapit (MLM), we considered six principal components, which we chose from the previously described structure analysis. The LFMM v2 method implements a Latent Factor Mixed Model that estimates unknown confounding factors (the latent factors) jointly using the genotypic and phenotypic matrices. For this specific model, the estimation of latent factors was performed using both the phenotypic data (i.e., the climate variable in consideration) and a genotypic matrix of SNPs with a MAF higher than $20 \%$. Once the GWAS step was performed, we used a $\mathrm{FDR}^{110}$ approach to estimate the significance of the associations for each analysis, retaining a FDR threshold of 5\%, resulting in a list of candidate positions for each variable and each GWAS method. We then considered a genomic window of $50 \mathrm{~kb}$ upstream and $50 \mathrm{~kb}$ downstream for each position as a candidate region, which we denominated quantitative trait locus (QTL). When two QTLs overlapped, they were combined.

To test SNP associations to ethnolinguistic groups, we used the PLINK software v1. $90^{111}$. We filtered out the SNPs with MAF below 5\%, more than $10 \%$ missing data, and in LD, resulting in 897,796 SNPs. The association of SNPs with each ethnic and linguistic group was tested by a logistic regression model assuming additive genetic effects, using three principal components as covariates to control for population stratification. Significant associations were identified above the threshold of $p$ value $=1 \mathrm{e}^{-05}$. Manhattan plots were plotted to display the associations using the qqman $\mathrm{R}$ package v0.1.4 $4^{112}$. GO terms for all fonio protein sequences were assigned using the DeepGOPlus model [https://github.com/bioontology-research-group/deepgoplus $]^{113}$. We only considered GO labels that have a confident score $>0.3$. Enrichment analysis for GO biological processes, cellular components, and molecular functions of the genes of interests from climate association analyses was performed using the Fisher exact tests implemented in $\mathrm{R}$ package topGO v3.36.0114 (algorithm = 'weight01'). We reported GO terms with enrichment $p$ value $<0.05$ for each GO-term category.

\section{Demographic history reconstruction. A Sequentially Markovian Coalescent-} based approach ${ }^{115,116}$ was used to infer past evolution of the effective population size of D. exilis and D. longiflora. This analysis was made with the smc $++{ }^{117}$ software and additional customized scripts [https://github.com/Africrop/ fonio_smcpp]. We excluded SNPs that were not usable (e.g. repeated regions) according to msmc-tools advices [https://github.com/stschiff/msmc-tools]. Analysis was done with all nine individuals from the genetically closest group of $D$. longiflora and also with the six groups of $D$. exilis based on the genetic structure The $s m c++$ vcf $2 s m c$ tools was used to generate the input file and smc ++ inference was performed for the different sets of genotypes using the $\mathrm{smc}++\mathrm{cv}$ command, considering 200 and 25,000 generations lower and upper time points boundaries, respectively. All other options were set to default. We considered a generation time of one year and a mutation rate of $6.5 \times 10^{-9}$. Different sets of distinguished lineages were considered for the different sets of individuals (all the samples were considered as distinguished). Graphical representation was made using the ggplot2 v3.3.2 $2^{68}$ package for R.

Genome scanning for selection signals. Detection of selection was performed independently with three different methods: (1) Weir and Cockerham $F_{\mathrm{ST}}$; (2) nucleotide diversity ratios $(\pi)$ were calculated between $D$. exilis and $D$. longiflora using VCFtools v0.1.1799 with sliding windows of $50 \mathrm{~kb}$ every $10 \mathrm{~kb}$; (3) SweeD v3.3.1 ${ }^{39}$ was used on $D$. exilis to detect signatures of selective sweeps based on the composite likelihood ratio (CLR) test within non-overlapping intervals of $3000 \mathrm{bp}$ along each chromosome. We grouped CLR peak positions into one common window if two or several peaks were $<100 \mathrm{~kb}$ apart. Candidate genomic regions were selected for each method based on top $1 \%$ values. A list comprising 34 wellcharacterized domestication genes (Supplementary Data 8) from major crops was selected and compared by BLAST v2.6.0 against gene models and the genome assembly of $D$. exilis accession CM05836. Each putative orthologous gene was validated by local alignment using ClustalW v2.190. We considered the genes as orthologous when $\geq 70 \%$ of the coding sequence (CDS) length hit the genomic sequence of $D$. exilis with at least $70 \%$ identity. Validated orthologous genes were crossed with genomic regions under selection using bedtools v2.28.0118. To assess the effect of $\Delta D e S h 1$ on seeds shattering, fonio panicles with three racemes were collected from mature plants and placed in $15 \mathrm{ml}$ tubes. Individual tubes were 
shaken using a Geno Grinder tissue homogenizer (SPEX SamplePrep, Metuchen, NJ) at $1350 \mathrm{rpm}$ speed for $20 \mathrm{~s}$ and the percentage of shattering was calculated. ANOVA test was performed with the MVApp [https://mvapp.kaust.edu.sa/] ${ }^{119}$. Two specific primer pairs were designed to confirm the $60 \mathrm{~kb}$ deletion. The first primer pair amplifies-1 kb fragment within the DeSh1-9A gene (forward primer 5'GTGACCATGCCAAGCAGGCG-3'; reverse primer 5'-GCTAGCTTGAGGTA TTTACGG- $3^{\prime}$ ). The second primer pair was designed in the flanking region of the deletion and was used to amplify $\sim 750 \mathrm{bp}$ fragment in accessions that carry the deletion (forward primer 5'-GCCGTATATAGTTGCCGCATCA-3'; reverse primer 5'-CCTACCGTTAGATCCGTGCGGA-3').

Reporting summary. Further information on research design is available in the Nature Research Reporting Summary linked to this article.

\section{Data availability}

Data supporting the findings of this work are available within the paper and its Supplementary Information files. A reporting summary for this Article is available as a Supplementary Information file. The datasets generated and analyzed during the current study are available from the corresponding author upon request. The raw sequencing data used for de novo whole-genome assembly, the raw bionano map, the CM05836 genome assembly, the RNA-seq data for the annotation and the 183 re-sequenced accessions of $D$. exilis and $D$. longiflora for the population genomics analysis are available on EBI-ENA under the study number PRJEB36539 [https://www.ebi.ac.uk/ena/browser/ view/PRJEB36539]. The annotation of the CM05836 genome, the Tritex assembly, the probes for the chromosome painting experiment, the gene ontology annotation, the VCF file and DeSh1 phenotyping data are available on the DRYAD database [https://oi.org/ 10.5061/dryad.2v6wwpzj0]. The plant coding sequences [https://plants.ensembl.org] and [https://genomevolution.org/coge/], the UniProt/SwissProt database (Release 2019_08[https://www.uniprot.org/]), the embryophyta_odb9 BUSCO dataset [https://buscoarchive.ezlab.org/v2/datasets/embryophyta_odb9.tar.gz], the Ethnologue version 16 language [https://www.ethnologue.com/]), and WorldClim version 1.4 [https://www worldclim.org/data/v1.4/worldclim14.html] databases were downloaded from source for data analyses. Source data are provided with this paper.

\section{Code availability}

Custom scripts for the SNP calling are available on GitHub: [https://github.com/ IBEXCluster/IBEX-SNPcaller]. Custom scripts used to launch the smc ++ analysis for fonio genome resequencing data are available on GitHub: [https://github.com/Africrop/ fonio_smcpp].

Received: 13 April 2020; Accepted: 16 August 2020;

Published online: 08 September 2020

\section{References}

1. Hickey, L. T. et al. Breeding crops to feed 10 billion. Nat. Biotechnol. 37, 744-754 (2019).

2. Tena, G. Sequencing forgotten crops. Nat. Plants 5, 5 (2019).

3. National Academies of Sciences Engineering and Medicine. Breakthroughs to Advance Food and Agricultural Research by 2030 (The National Academies Press, Washington, 2019).

4. FAO. The State of Agricultural Commodity Markets 2018. Agricultural Trade, Climate Change and Food Security (FAO, Rome, 2018).

5. Dalin, C., Wada, Y., Kastner, T. \& Puma, M. J. Groundwater depletion embedded in international food trade. Nature 543, 700-704 (2017).

6. Fernie, A. R. \& Yan, J. De novo domestication: an alternative route toward new crops for the future. Mol. Plant 12, 615-631 (2019).

7. Tanksley, S. D. \& McCouch, S. R. Seed banks and molecular maps: unlocking genetic potential from the wild. Science 277, 1063-1066 (1997).

8. Gruber, K. Agrobiodiversity: the living library. Nature 544, S8-S10 (2017).

9. Kistler, L. et al. Multiproxy evidence highlights a complex evolutionary legacy of maize in South America. Science 362, 1309-1313 (2018).

10. Wing, R. A., Purugganan, M. D. \& Zhang, Q. F. The rice genome revolution: from an ancient grain to Green Super Rice. Nat. Rev. Genet. 19, 505-517 (2018).

11. Eshed, Y. \& Lippman, Z. B. Revolutions in agriculture chart a course for targeted breeding of old and new crops. Science 366, eaax0025 (2019).

12. Kantar, M. B. \& Runck, B. Take a walk on the wild side. Nat. Clim. Change 9, 731-732 (2019).

13. Dawson, I. K. et al. The role of genetics in mainstreaming the production of new and orphan crops to diversify food systems and support human nutrition. New Phytol. 224, 37-54 (2019)

14. Pironon, $\mathrm{S}$. et al. Potential adaptive strategies for 29 sub-Saharan crops under future climate change. Nat. Clim. Change 9, 758-763 (2019).
15. Wallace, J. G., Rodgers-Melnick, E. \& Buckler, E. S. On the road to breeding 4.0: unraveling the good, the bad, and the boring of crop quantitative genomics. Annu. Rev. Genet. 52, 421-444 (2018).

16. Chen, K., Wang, Y., Zhang, R., Zhang, H. \& Gao, C. CRISPR/Cas genome editing and precision plant breeding in agriculture. Annu. Rev. Plant Biol. 70, 667-697 (2019).

17. Barnaud, A. et al. High selfing rate inferred for white fonio [Digitaria exilis (Kippist.) Stapf] reproductive system opens up opportunities for breeding programs. Genet. Resour. Crop Evol. 64, 1485-1490 (2017).

18. Ayenan, M. A. T., Sodedji, K. A. F., Nwankwo, C. I., Olodo, K. F. \& Alladassi, M. E. B. Harnessing genetic resources and progress in plant genomics for fonio (Digitaria spp.) improvement. Genet. Resour. Crop Evol. 65, 373-386 (2018).

19. Cruz, J. F. \& Beavogui, F. Fonio, an African Cereal (CIRAD, France, 2016).

20. Adoukonou-Sagbadja, H., Wagner, C., Ordon, F. \& Friedt, W. Reproductive system and molecular phylogenetic relationships of fonio millets (Digitaria spp., Poaceae) with some polyploid wild relatives. Trop. Plant Biol. 3, 240-251 (2010).

21. Abdul, S. D. \& Jideani, A. I. O. Fonio (Digitaria spp.) breeding. In Advances in Plant Breeding Strategies: Cereals (eds Al-Khayri, J. M., Jain, S. M. \& Johnson, D. V.) 47-81 (Springer, 2019)

22. Adoukonou-Sagbadja, H. et al. Flow cytometric analysis reveals different nuclear DNA contents in cultivated Fonio (Digitaria spp.) and some wild relatives from West-Africa. Plant Syst. Evol. 267, 163-176 (2007).

23. Avni, R. et al. Wild emmer genome architecture and diversity elucidate wheat evolution and domestication. Science 357, 93-97 (2017).

24. Edger, P. P. et al. Origin and evolution of the octoploid strawberry genome Nat. Genet. 51, 541-547 (2019).

25. Springer, N. M. et al. The maize W22 genome provides a foundation for functional genomics and transposon biology. Nat. Genet. 50, 1282-1288 (2018).

26. Han, Y. H., Zhang, T., Thammapichai, P., Weng, Y. Q. \& Jiang, J. M. Chromosome-specific painting in Cucumis species using bulked oligonucleotides. Genetics 200, 771-779 (2015).

27. Monat, C. et al. TRITEX: chromosome-scale sequence assembly of Triticeae genomes with open-source tools. Genome Biol. 20, 284 (2019).

28. Bennetzen, J. L. et al. Reference genome sequence of the model plant Setaria. Nat. Biotechnol. 30, 555-561 (2012).

29. Tang, H. Disentangling a polyploid genome. Nat. Plants 3, 688-689 (2017).

30. Suguiyama, V. F., Vasconcelos, L. A. B., Rossi, M. M., Biondo, C. \& de Setta $\mathrm{N}$. The population genetic structure approach adds new insights into the evolution of plant LTR retrotransposon lineages. PLoS ONE 14, e0214542 (2019).

31. International Wheat Genome Sequencing Consortium. Shifting the limits in wheat research and breeding through a fully annotated and anchored reference genome sequence. Science 361, eaar7191 (2018).

32. Ramirez-Gonzalez, R. H. et al. The transcriptional landscape of polyploid wheat. Science 361, eaar6089 (2018)

33. Bird, K. A., VanBuren, R., Puzey, J. R. \& Edger, P. P. The causes and consequences of subgenome dominance in hybrids and recent polyploids. New Phytol. 220, 87-93 (2018).

34. Schnable, J. C., Springer, N. M. \& Freeling, M. Differentiation of the maize subgenomes by genome dominance and both ancient and ongoing gene loss. Proc. Natl Acad. Sci. USA 108, 4069-4074 (2011).

35. Shi, J. P. et al. Chromosome conformation capture resolved near complete genome assembly of broomcorn millet. Nat. Commun. 10, 464 (2019).

36. Clément, J. \& Leblanc, J. M. Collecte IBPGR-ORSTOM de 1977 au Togo (Catalogue ORSTOM, 1984)

37. Ramu, P. et al. Cassava haplotype map highlights fixation of deleterious mutations during clonal propagation. Nat. Genet. 49, 959-963 (2017)

38. Patwari, P. et al. Surface wax esters contribute to drought tolerance in Arabidopsis. Plant J. 98, 727-744 (2019).

39. Pavlidis, P., Zivkovic, D., Stamatakis, A. \& Alachiotis, N. SweeD: Likelihoodbased detection of selective sweeps in thousands of genomes. Mol. Biol. Evol. 30, 2224-2234 (2013)

40. Li, Y. et al. Natural variation in GS5 plays an important role in regulating grain size and yield in rice. Nat. Genet. 43, 1266-1269 (2011)

41. Lin, Z. W. et al. Parallel domestication of the Shattering1 genes in cereals. Nat. Genet. 44, 720-724 (2012).

42. Wang, M. H. et al. The genome sequence of African rice (Oryza glaberrima) and evidence for independent domestication. Nat. Genet. 46, 982-988 (2014).

43. VanBuren, R. et al. Exceptional subgenome stability and functional divergence in the allotetraploid Ethiopian cereal teff. Nat. Commun. 11, 884 (2020).

44. Cubry, P. et al. The rise and fall of African rice cultivation revealed by analysis of 246 new genomes. Curr. Biol. 28, 2274-2282 (2018).

45. Liang, Z. et al. Whole-genome resequencing of 472 Vitis accessions for grapevine diversity and demographic history analyses. Nat. Commun. 10, 1190 (2019). 
46. Blench, R. M. Vernacular names for African millets and other minor cereals and their significance for agricultural history. Archaeol. Anthropol. Sci. 8, 1-8 (2016).

47. Adoukonou-Sagbadja, H., Dansi, A., Vodouhe, R. \& Akpagana, K. Collecting fonio (Digitaria exilis Kipp. Stapf, D. iburua Stapf) landraces in Togo. Plant Genet. Resour. Newsl. 139, 63-67 (2004).

48. Meyer, R. S. \& Purugganan, M. D. Evolution of crop species: genetics of domestication and diversification. Nat. Rev. Genet. 14, 840-852 (2013).

49. Barnaud, A. et al. Development of nuclear microsatellite markers for the fonio, Digitaria exilis (Poaceae), an understudied West African cereal. Am. J. Bot. 99, E105-E107 (2012).

50. Doležel, J., Greilhuber, J. \& Suda, J. Estimation of nuclear DNA content in plants using flow cytometry. Nat. Protoc. 2, 2233-2244 (2007).

51. Doležel, J., Bartoš, J., Voglmayr, H. \& Greilhuber, J. Nuclear DNA content and genome size of trout and human. Cytometry 51A, 127-128 (2003).

52. Jackman, S. D. et al. Tigmint: correcting assembly errors using linked reads from large molecules. BMC Bioinforma. 19, 393 (2018).

53. Coombe, L. et al. ARKS: chromosome-scale scaffolding of human genome drafts with linked read kmers. BMC Bioinforma. 19, 234 (2018).

54. Warren, R. L. et al. LINKS: scalable, alignment-free scaffolding of draft genomes with long reads. GigaScience 4, 35 (2015)

55. Li, H. \& Durbin, R. Fast and accurate long-read alignment with Burrows-Wheeler transform. Bioinformatics 26, 589-595 (2010).

56. Dudchenko, O. et al. De novo assembly of the Aedes aegypti genome using HiC yields chromosome-length scaffolds. Science 356, 92-95 (2017).

57. Novak, P., Neumann, P., Pech, J., Steinhaisl, J. \& Macas, J. RepeatExplorer: a Galaxy-based web server for genome-wide characterization of eukaryotic repetitive elements from next-generation sequence reads. Bioinformatics 29, 792-793 (2013).

58. Untergasser, A. et al. Primer3-new capabilities and interfaces. Nucleic Acids Res. 40, e115 (2012).

59. Šimoníková, D. et al. Chromosome painting facilitates anchoring reference genome sequence to chromosomes in situ and integrated karyotyping in banana (Musa Spp.). Front. Plant Sci. 10, 1503 (2019).

60. Ellinghaus, D., Kurtz, S. \& Willhoeft, U. LTRharvest, an efficient and flexible software for de novo detection of LTR retrotransposons. BMC Bioinforma. 9, 18 (2008).

61. Xu, Z. \& Wang, H. LTR_FINDER: an efficient tool for the prediction of fulllength LTR retrotransposons. Nucleic Acids Res. 35, W265-W268 (2007).

62. Ou, S. J. \& Jiang, N. LTR_retriever: a highly accurate and sensitive program for identification of long terminal repeat retrotransposons. Plant Physiol. 176, 1410-1422 (2018).

63. James, B. T., Luczak, B. B. \& Girgis, H. Z. MeShClust: an intelligent tool for clustering DNA sequences. Nucleic Acids Res. 46, e83 (2018).

64. Sonnhammer, E. L. \& Durbin, R. A dot-matrix program with dynamic threshold control suited for genomic DNA and protein sequence analysis. Gene 167, GC1-GC10 (1995).

65. Sievers, F. et al. Fast, scalable generation of high-quality protein multiple sequence alignments using Clustal Omega. Mol. Syst. Biol. 7, 539 (2011).

66. Knaus, B. J. \& Grunwald, N. J. VCFR: a package to manipulate and visualize variant call format data in R. Mol. Ecol. Resour. 17, 44-53 (2017).

67. Jombart, $\mathrm{T}$. adegenet: a $\mathrm{R}$ package for the multivariate analysis of genetic markers. Bioinformatics 24, 1403-1405 (2008).

68. Wickham, H. ggplot2 - Elegant Graphics for Data Analysis (Springer International Publishing, 2016)

69. Ma, J. X. \& Bennetzen, J. L. Rapid recent growth and divergence of rice nuclear genomes. Proc. Natl Acad. Sci. USA 101, 12404-12410 (2004).

70. Edgar, R. C. Search and clustering orders of magnitude faster than BLAST. Bioinformatics 26, 2460-2461 (2010).

71. Cantarel, B. L. et al. MAKER: an easy-to-use annotation pipeline designed for emerging model organism genomes. Genome Res. 18, 188-196 (2008).

72. Kopylova, E., Noe, L. \& Touzet, H. SortMeRNA: fast and accurate filtering of ribosomal RNAs in metatranscriptomic data. Bioinformatics 28, 3211-3217 (2012).

73. Bolger, A. M., Lohse, M. \& Usadel, B. Trimmomatic: a flexible trimmer for Illumina sequence data. Bioinformatics 30, 2114-2120 (2014).

74. Dobin, A. et al. STAR: ultrafast universal RNA-seq aligner. Bioinformatics 29, 15-21 (2013)

75. Pertea, M. et al. StringTie enables improved reconstruction of a transcriptome from RNA-seq reads. Nat. Biotechnol. 33, 290-295 (2015).

76. The Arabidopsis Genome Initiative. Analysis of the genome sequence of the flowering plant Arabidopsis thaliana. Nature 408, 796-815 (2000).

77. Paterson, A. H. et al. The Sorghum bicolor genome and the diversification of grasses. Nature 457, 551-556 (2009).

78. Schnable, P. S. et al. The B73 maize genome: complexity, diversity, and dynamics. Science 326, 1112-1115 (2009).

79. International Rice Genome Sequencing Project. The map-based sequence of the rice genome. Nature 436, 793-800 (2005).
80. Altschul, S. F. et al. Gapped BLAST and PSI-BLAST: a new generation of protein database search programs. Nucleic Acids Res. 25, 3389-3402 (1997)

81. Slater, G. S. \& Birney, E. Automated generation of heuristics for biological sequence comparison. BMC Bioinforma. 6, 31 (2005).

82. Borodovsky, M. \& Lomsadze, A. Eukaryotic gene prediction using GeneMark hmm-E and GeneMark-ES. Curr. Protoc. Bioinforma. 35, 4.6.1-4.6.10 (2011)

83. Korf, I. Gene finding in novel genomes. BMC Bioinforma. 5, 59 (2004)

84. Stanke, M. \& Waack, S. Gene prediction with a hidden Markov model and a new intron submodel. Bioinformatics 19, ii215-ii225 (2003)

85. Lovell, J. T. et al. The genomic landscape of molecular responses to natural drought stress in Panicum hallii. Nat. Commun. 9, 5213 (2018).

86. The International Brachypodium Initiative. Genome sequencing and analysis of the model grass Brachypodium distachyon. Nature 463, 763-768 (2010)

87. Mascher, M. et al. A chromosome conformation capture ordered sequence of the barley genome. Nature 544, 427-433 (2017).

88. Luo, M. C. et al. Genome sequence of the progenitor of the wheat D genome Aegilops tauschii. Nature 551, 498-502 (2017).

89. Wang, Y. P. et al. MCScanX: a toolkit for detection and evolutionary analysis of gene synteny and collinearity. Nucleic Acids Res. 40, e49 (2012).

90. Thompson, J. D., Higgins, D. G. \& Gibson, T. J. Clustal-W - Improving the sensitivity of progressive multiple sequence alignment through sequence weighting, position-specific gap penalties and weight matrix choice. Nucleic Acids Res. 22, 4673-4680 (1994).

91. Yang, Z. H. PAML 4: phylogenetic analysis by maximum likelihood. Mol. Biol. Evol. 24, 1586-1591 (2007).

92. Gaut, B. S., Morton, B. R., McCaig, B. C. \& Clegg, M. T. Substitution rate comparisons between grasses and palms: synonymous rate differences at the nuclear gene Adh parallel rate differences at the plastid gene rbcL. Proc. Nat Acad. Sci. USA 93, 10274-10279 (1996).

93. Hu, F., Lin, Y. \& Tang, J. MLGO: phylogeny reconstruction and ancestral inference from gene-order data. BMC Bioinforma. 15, 354 (2014).

94. Ren, L., Huang, W. \& Cannon, S. B. Reconstruction of ancestral genome reveals chromosome evolution history for selected legume species. New Phytol. 223, 2090-2103 (2019).

95. Li, B. \& Dewey, C. N. RSEM: accurate transcript quantification from RNASeq data with or without a reference genome. BMC Bioinforma. 12, 323 (2011).

96. Li, H. et al. The Sequence Alignment/Map format and SAMtools. Bioinformatics 25, 2078-2079 (2009).

97. McKenna, A. et al. The Genome Analysis Toolkit: a MapReduce framework for analyzing next-generation DNA sequencing data. Genome Res. 20, 1297-1303 (2010).

98. Van der Auwera, G. A. et al. From FastQ data to high confidence variant calls: the Genome Analysis Toolkit best practices pipeline. Curr. Protoc. Bioinforma. 43, 1110 1-11 1033 (2013).

99. Danecek, P. et al. The variant call format and VCFtools. Bioinformatics 27, 2156-2158 (2011)

100. Cingolani, P. et al. A program for annotating and predicting the effects of single nucleotide polymorphisms, SnpEff: SNPs in the genome of Drosophila melanogaster strain w(1118); iso-2; iso-3. Fly 6, 80-92 (2012).

101. Frichot, E. \& Francois, O. LEA: an R package for landscape and ecological association studies. Methods Ecol. Evol. 6, 925-929 (2015).

102. Nychka, D., Furrer, R., Paige, J. \& Sain, S. fields: Tools for Spatial Data. Retrieved from https://cran.r-project.org/package=fields (2017).

103. Cubry, P., Vigouroux, Y. \& Francois, O. The empirical distribution of singletons for geographic samples of DNA sequences. Front. Genet. 8, 139 (2017)

104. Zhang, C., Dong, S. S., Xu, J. Y., He, W. M. \& Yang, T. L. PopLDdecay: a fas and effective tool for linkage disequilibrium decay analysis based on variant call format files. Bioinformatics 35, 1786-1788 (2019)

105. Hijmans, R. J., Cameron, S. E., Parra, J. L., Jones, P. G. \& Jarvis, A. Very high resolution interpolated climate surfaces for global land areas. Int. J. Climatol. 25, 1965-1978 (2005).

106. Cubry, P. et al. Genome wide association study pinpoints key agronomic QTLs in African rice Oryza glaberrima. Preprint at https://doi.org/10.1101/ 2020.01.07.897298 (2020)

107. Kang, H. M. et al. Efficient control of population structure in model organism association mapping. Genetics 178, 1709-1723 (2008)

108. Lipka, A. E. et al. GAPIT: genome association and prediction integrated tool. Bioinformatics 28, 2397-2399 (2012).

109. Caye, K., Jumentier, B., Lepeule, J. \& Francois, O. LFMM 2: fast and accurate inference of gene-environment associations in genome-wide studies. Mol. Biol. Evol. 36, 852-860 (2019).

110. Benjamini, Y. \& Hochberg, Y. Controlling the false discovery rate: a practical and powerful approach to multiple testing. J. R. Stat. Soc. Ser. (Methdol.) 57, 289-300 (1995).

111. Purcell, S. et al. PLINK: A tool set for whole-genome association and population-based linkage analyses. Am. J. Hum. Genet. 81, 559-575 (2007). 
112. Turner, S. D. qqman: an R package for visualizing GWAS results usingQ-Q and Manhattan plots. J. Open Source Softw. 3, 731 (2018).

113. Kulmanov, M. \& Hoehndorf, R. DeepGOPlus: improved protein function prediction from sequence. Bioinformatics 36, 422-429 (2020).

114. Alexa, A., Rahnenfuhrer, J. \& Lengauer, T. Improved scoring of functional groups from gene expression data by decorrelating GO graph structure. Bioinformatics 22, 1600-1607 (2006).

115. Li, H. \& Durbin, R. Inference of human population history from individual whole-genome sequences. Nature 475, 493-496 (2011).

116. Schiffels, S. \& Durbin, R. Inferring human population size and separation history from multiple genome sequences. Nat. Genet. 46, 919-925 (2014).

117. Terhorst, J., Kamm, J. A. \& Song, Y. S. Robust and scalable inference of population history froth hundreds of unphased whole genomes. Nat. Genet. 49, 303-309 (2017).

118. Quinlan, A. R. \& Hall, I. M. BEDTools: a flexible suite of utilities for comparing genomic features. Bioinformatics 26, 841-842 (2010).

119. Julkowska, M. M. et al. MVApp-Multivariate analysis application for streamlined data analysis and curation. Plant Physiol. 180, 1261-1276 (2019).

\section{Acknowledgements}

We are grateful to Vinicius M. Lube, Samantha Bazan, Ablaye Ngom, Marie Piquet, Hélène Adam, and Carole Gauron for their technical assistance in Herbarium sampling and photography. Cirad herbarium samples of Digitaria longiflora were provided by Samantha Bazan (ALF [http://publish.plantnet-project.org/]). We thank Soukeye Conde for valuable insight, funded by the project Cultivar, reference ANR-16-IDEX-0006, through the Investissements d'avenir program (Labex Agro: ANR-10-LABX-0001-01). This work was supported by the CIRAD-UMR AGAP and IRD UMR DIADE HPC Data Center of the South Green Bioinformatics platform [http://www.southgreen.fr/] and the Center for Desert Agriculture of the King Abdullah University of Science and Technology (KAUST). D.S., J.C., E.H. and J.D. were in part supported by the European Regional Development Fund OPVVV project 'Plants as a tool for sustainable development' number CZ.02.1.01/0.0/0.0/16_019/0000827.

\section{Author contributions}

M.A., A.B., Y.V., and S.G.K. designed research. N.K. and M.A. established the SNP calling pipeline. J.B., M.C., and L.Z. performed molecular analyses and constructed sequencing libraries. M.A., H.I.A., P.C., L.G., N.S., and T.W. performed bioinformatics analyses. H.I.A, P.C. and Y.P. performed association studies. M.A., H.I.A., P.C., Y.V.,
C.B., A.B., and S.G.K. interpret results. D.S., J.C., E.H., and J.D. performed flowcytometry and chromosome painting. S.A., S.C., and H.B. constructed and analyzed the CM05836 optical map. Y.P. and H.I.A carried out phenotypic data collection and analyses. J.J.W., M.G., N.A.K., C.L., S.C., S.V., and C.B. contributed biological materials. M.A., H.I.A., and S.G.K. wrote the paper with substantial inputs from P.C., Y.V., and A.B. All authors have read and approved the manuscript.

\section{Competing interests}

The authors declare no competing interests.

\section{Additional information}

Supplementary information is available for this paper at https://doi.org/10.1038/s41467020-18329-4.

Correspondence and requests for materials should be addressed to A.B. or S.G.K.

Peer review information Nature Communications thanks the anonymous reviewers for their contribution to the peer review of this work. Peer reviewer reports are available.

Reprints and permission information is available at http://www.nature.com/reprints

Publisher's note Springer Nature remains neutral with regard to jurisdictional claims in published maps and institutional affiliations.

(c) (i) Open Access This article is licensed under a Creative Commons Attribution 4.0 International License, which permits use, sharing, adaptation, distribution and reproduction in any medium or format, as long as you give appropriate credit to the original author(s) and the source, provide a link to the Creative Commons license, and indicate if changes were made. The images or other third party material in this article are included in the article's Creative Commons license, unless indicated otherwise in a credit line to the material. If material is not included in the article's Creative Commons license and your intended use is not permitted by statutory regulation or exceeds the permitted use, you will need to obtain permission directly from the copyright holder. To view a copy of this license, visit http://creativecommons.org/ licenses/by/4.0/.

(c) The Author(s) 2020 